\title{
JOINT DIAMONDS AND LAVER DIAMONDS
}

\author{
MIHA E. HABIČ
}

\begin{abstract}
The concept of jointness for guessing principles, specifically $\diamond_{\kappa}$ and various Laver diamonds, is introduced. A family of guessing sequences is joint if the elements of any given sequence of targets may be simultaneously guessed by the members of the family. While equivalent in the case of $\nabla_{\kappa}$, joint Laver diamonds are nontrivial new objects. We give equiconsistency results for most of the large cardinals under consideration and prove sharp separations between joint Laver diamonds of different lengths in the case of $\theta$-supercompact cardinals.
\end{abstract}

\section{INTRODUCTION}

The notion of a Laver function, introduced for supercompact cardinals in [16], is a powerful strengthening of the usual $\diamond$-principle to the large cardinal setting. It is based on the observation that a large variety of large cardinal properties give rise to different notions of a "large" set, intermediate between stationary and club, and these are then used to provide different guessing principles, where we require that the sequence guesses correctly on these "large" sets. This is usually recast in terms of elementary embeddings or extensions (if the large cardinal in question admits such a characterization), using various ultrapower constructions. For example, in the case of a supercompact cardinal $\kappa$, the usual definition states that a Laver function for $\kappa$ is a function $\ell: \kappa \rightarrow V_{\kappa}$ such that for any $\theta$ and any $a \in H_{\theta^{+}}$there is a $\theta$-supercompactness embedding $j: V \rightarrow M$ with critical point $\kappa$ such that $j(\ell)(\kappa)=a$ (this ostensibly second order definition can be rendered in first order language by replacing the quantification over arbitrary embeddings with quantification over ultrapowers by measures on $\mathcal{P}_{\kappa}(\theta)$, as in Laver's original account). In this example, Łoś's theorem tells us that the set of $\alpha<\kappa$, for which $\ell(\alpha)$ codes an "initial segment" of $a$, is large, in the sense that it has measure 1 with respect to the normal measure on $\kappa$ derived from $j$.

Laver functions for other large cardinals were later defined by Gitik and Shelah [9], Corazza [5], Hamkins [11], and others. The term Laver diamonds has been suggested to more strongly underline the connection between the large and small cardinal versions.

2010 Mathematics Subject Classification. 03E55, 03E35.

Key words and phrases. Laver functions, joint Laver functions, joint diamond sequences.

The author is grateful to the anonymous referee for their numerous suggestions, which greatly improved the presentation in the paper.

The material presented in this paper is part of the author's doctoral dissertation, completed at the Graduate Center, CUNY, under the supervision of Joel David Hamkins.

The author was partly supported by the ESIF, EU Operational Programme Research, Development and Education, the International Mobility of Researchers in CTU project no. (CZ.02.2.69/0.0/0.0/16_027/0008465) at the Czech Technical University in Prague, and the joint FWF-GAČR grant no. 17-33849L: Filters, Ultrafilters and Connections with Forcing. 
In this paper we examine the notion of jointness for both ordinary and Laver diamonds. We shall give a simple example in Section 2; for now let us just say that a family of Laver diamonds is joint if they can guess their targets simultaneously and independently of one another. Section 2 also introduces some terminology that will ease later discussion. Sections 3 and 4 deal with the outright existence or at least the consistency of the existence of joint Laver sequences for supercompact and strong cardinals, respectively. Our results will show that in almost all cases the existence of a joint Laver sequence of maximal possible length is simply equiconsistent with the particular large cardinal. The exception are the $\theta$-strong cardinals where $\theta$ is a limit of small cofinality, for which we prove that additional strength is required for even the shortest joint sequences to exist. We also show that there are no nontrivial implications between the existence of joint Laver sequences of different lengths. Section 5 considers joint $\nabla_{\kappa}$-sequences and their relation to other known principles. Our main result there shows that, for a fixed $\kappa$, the principle $\nabla_{\kappa}$ is simply equivalent to the existence of a joint $\nabla_{\kappa}$-sequence of any possible length.

We shall list open questions wherever they arise in the course of exposition.

\section{JoINTNESS: A MOTIVATING EXAMPLE}

All of the large cardinals we will be dealing with in this paper are characterized by the existence of elementary embeddings of the universe into inner models which have that cardinal as their critical point. We can thus speak of embeddings associated to a measurable, a $\theta$-strong, a 17-huge cardinal, and so forth. At this stage we do not insist that these embeddings are any kind of ultrapower embedding, or even definable, so this whole introductory discussion should take place in an appropriate second-order setting. Since the definitions of (joint) Laver diamonds for these various large cardinals are quite similar, we give the following general definition as a framework to aid future exposition.

Definition 1. Let $j$ be an elementary embedding of the universe witnessing the largeness of its critical point $\kappa$ (a measurable or a $(\kappa+2)$-strongness embedding, for instance) and let $\ell$ be a function defined on $\kappa$. We say that a set $a$, the target, is guessed by $\ell$ via $j$ if $j(\ell)(\kappa)=a$.

If $A$ is a set or a definable class, say that $\ell$ is an A-guessing Laver function (or Laver diamond) if for any $a \in A$ there is an embedding $j$, witnessing the largeness of $\kappa$, such that $\ell$ guesses $a$ via $j$. If there is an $A$-guessing Laver function for $\kappa$, we shall say that $\triangle_{\kappa}(A)$ holds. ${ }^{1}$

To simplify the terminology even more, we shall associate to each type of large cardinal considered, a default set of targets $A$ (for example, when talking about a measurable cardinal $\kappa$, we will be predominantly interested in targets from $H_{\kappa^{+}}$). In view of this, whenever we neglect the mention of a particular class of targets, these default targets will be intended.

We will often specify the type of large cardinal embeddings we have in mind explicitly, by writing $\triangle_{\kappa}^{\text {meas }}$, or $\Delta_{\kappa}^{\theta \text {-sc }}$, or similar. This is to avoid ambiguity; for example, we could conceivably start with a supercompact cardinal $\kappa$ but only be interested in its measurable Laver functions. Even so, to keep the notation as

\footnotetext{
${ }^{1}$ Different notation has been used by different authors to denote the existence of a Laver function. We chose here to follow Hamkins [11].
} 
unburdened as possible, we may sometimes omit the specific large cardinal property under consideration when it is clear from context.

As a further complication, the stated definition of an $A$-guessing Laver function is second-order, since we are quantifying over all possible embeddings $j$. This is unavoidable for arbitrary $A$. However, the default sets of targets we shall be working with are chosen in such a way that standard factoring arguments allow us to restrict our attention to ultrapower embeddings (by measures or extenders). The most relevant definitions of Laver functions can therefore be recast in first-order language in the usual way.

Given the concept of a Laver diamond for a large cardinal $\kappa$, we might ask when two Laver functions are different and how many distinct ones can $\kappa$ carry. It is clear that the guessing behaviour of these functions is determined by their restrictions to large (in the sense of an appropriate large-cardinal measure) sets; in other words, $j(\ell)(\kappa)$ and $j\left(\ell^{\prime}\right)(\kappa)$ equal one another if $\ell$ and $\ell^{\prime}$ only differ on a small (nonstationary, say) subset of their domain. We definitely do not want to count these functions as distinct: they cannot even guess distinct targets! Instead, what we want are Laver functions whose targets, under a single embedding $j$, can be chosen completely independently. Let us illustrate this situation with a simple example.

Suppose $\ell: \kappa \rightarrow V_{\kappa}$ is a supercompactness Laver function as defined in the introduction. We can then define two functions $\ell_{0}, \ell_{1}$ by letting $\ell_{0}(\xi)$ and $\ell_{1}(\xi)$ be the first and second components, respectively, of $\ell(\xi)$, if this happens to be an ordered pair. These two are then easily seen to be Laver functions themselves, but have the additional property that, given any pair of targets $a_{0}, a_{1}$, there is a single supercompactness embedding $j$ such that $j\left(\ell_{0}\right)(\kappa)=a_{0}$ and $j\left(\ell_{1}\right)(\kappa)=a_{1}$ (just the one that makes $\left.j(\ell)(\kappa)=\left(a_{0}, a_{1}\right)\right)$. This additional trait, where two Laver functions are, in a sense, enmeshed, we call jointness.

Definition 2. Let $A$ be a set or a definable class and let $\kappa$ be a cardinal with a notion of $A$-guessing Laver function. A sequence $\vec{\ell}=\left\langle\ell_{\alpha} ; \alpha<\lambda\right\rangle$ of $A$-guessing Laver functions is an A-guessing joint Laver sequence if for any sequence $\vec{a}=$ $\left\langle a_{\alpha} ; \alpha<\lambda\right\rangle$ of targets from $A$ there is a single embedding $j$, witnessing the largeness of $\kappa$, such that each $\ell_{\alpha}$ guesses $a_{\alpha}$ via $j$. If there is an $A$-guessing joint Laver sequence of length $\lambda$ for $\kappa$, we shall say that $\triangle_{\kappa, \lambda}(A)$ holds.

In other words, a sequence of Laver diamonds is joint if, given any sequence of targets, these targets can be guessed simultaneously by their respective Laver diamonds.

We must be careful to distinguish between entire sequence being jointly Laver and its members being pairwise jointly Laver. It is not difficult to find examples of three (or four or even infinitely many) Laver functions that are pairwise joint but not fully so. For example, given two joint Laver functions $\ell_{0}$ and $\ell_{1}$, we might define $\ell_{2}(\xi)$ to be the symmetric difference of $\ell_{0}(\xi)$ and $\ell_{1}(\xi)$. It is easy to check that any two of these three functions can have their targets freely chosen, but the third one is uniquely determined by the other two.

Jointness also makes sense for ordinary diamond sequences, but needs to be formulated differently, since elementary embeddings do not (obviously) appear in that setting. Rather, we distil jointness for Laver diamonds into a property of certain ultrafilters and then apply this to more general filters and diamond sequences. We explore this further in Section 5 . 


\section{JOINT LAVER DIAMONDS FOR SUPERCOMPACT CARDINALS}

Definition 3. A function $\ell: \kappa \rightarrow V_{\kappa}$ is a $\theta$-supercompactness Laver function for $\kappa$ if it guesses elements of $H_{\theta^{+}}$via $\theta$-supercompactness embeddings with critical point $\kappa$. This also includes the case of $\kappa$ being measurable (as this is equivalent to it being $\kappa$-supercompact).

If $\kappa$ is fully supercompact, then a function $\ell: \kappa \rightarrow V_{\kappa}$ is a Laver function for $\kappa$ if it is a $\theta$-supercompactness Laver function for $\kappa$ for all $\theta$.

We shall say that $\Delta_{\kappa}^{\theta \text {-sc }}$ holds if there is a $\theta$-supercompactness Laver function for $\kappa$; in view of the definition just stated $H_{\theta+}$ is the default set of targets for these Laver functions, and so $\Delta_{\kappa}^{\theta \text {-sc }}$ is merely a synonym for $\Delta_{\kappa}^{\theta \text {-sc }}\left(H_{\theta^{+}}\right)$. Similarly, $\Delta_{\kappa}^{\text {meas }}$ will denote the existence of a measurable Laver function for $\kappa$ and is a synonym for $\Delta_{\kappa}^{\text {meas }}\left(H_{\kappa^{+}}\right)$. For fully supercompact cardinals $\kappa$, the existence of a supercompactness Laver function will be denoted by $\triangle_{\kappa}^{\mathrm{sc}}$, which should be read more precisely as $\triangle_{\kappa}^{\mathrm{sc}}(V)$.

While the definition of a $\theta$-supercompactness Laver function refers to arbitrary $\theta$ supercompactness embeddings, one can in fact work solely with embeddings arising from normal measures on $\mathcal{P}_{\kappa}(\theta)$. This is because any $\theta$-supercompactness embedding $j$ can be factored as $j=k \circ i$, where $i$ is the ultrapower by the induced normal measure on $\mathcal{P}_{\kappa}(\theta)$ and $k$ is an elementary embedding with critical point strictly above $\theta$. Since we are only interested in guessing targets from $H_{\theta^{+}}$by $\ell$, we get the same value whether we compute $j(\ell)(\kappa)$ or $i(\ell)(\kappa)$. In brief, if $\ell$ guesses a target in $H_{\theta^{+}}$via any $\theta$-supercompactness embedding, then it does so via a $\theta$ supercompactness ultrapower embedding. Moreover, in the joint setting, if several $\ell_{\alpha}$ guess their targets via a single $\theta$-supercompactness embedding, then they all guess their targets via a single $\theta$-supercompactness embedding as well.

Observe that there are at most $2^{\kappa}$ many $\theta$-supercompactness Laver functions for $\kappa$, since there are only $2^{\kappa}$ many functions $\kappa \rightarrow V_{\kappa}$. Since a joint Laver sequence cannot have the same function appear on two different coordinates (as they could never guess two different targets), this implies that $\lambda=2^{\kappa}$ is the largest cardinal for which there could possibly be a joint Laver sequence of length $\lambda$. Bounding from the other side, a single $\theta$-supercompactness Laver function, for some $\theta \leq 2^{\kappa}$, already yields a joint Laver sequence of length $\theta$.

Proposition 4. If $\triangle_{\kappa}^{\theta \text {-sc }}$ holds, then there is a $\theta$-supercompactness joint Laver sequence for $\kappa$ of length $\lambda=\min \left\{\theta, 2^{\kappa}\right\}$.

Proof. Let $\ell$ be a Laver function for $\kappa$, and fix a subset $I$ of $\mathcal{P}(\kappa)$ of size $\lambda$ and a bijection $f: \lambda \rightarrow I$. For $\alpha<\lambda$ define $\ell_{\alpha}: \kappa \rightarrow V_{\kappa}$ by $\ell_{\alpha}(\xi)=\ell(\xi)(f(\alpha) \cap \xi)$ if this makes sense and $\ell_{\alpha}(\xi)=\emptyset$ otherwise. We claim that $\left\langle\ell_{\alpha} ; \alpha<\lambda\right\rangle$ is a joint Laver sequence for $\kappa$.

To verify this, let $\vec{a}=\left\langle a_{\alpha} ; \alpha<\lambda\right\rangle$ be a sequence of elements of $H_{\theta^{+}}$. Then $\vec{a} \circ f^{-1} \in H_{\theta^{+}}$, so by assumption there is a $\theta$-supercompactness embedding $j: V \rightarrow$ $M$ such that $j(\ell)(\kappa)=\vec{a} \circ f^{-1}$. But now observe that, for any $\alpha<\lambda$,

$$
j\left(\ell_{\alpha}\right)(\kappa)=j(\ell)(\kappa)(j(f(\alpha)) \cap \kappa)=j(\ell)(\kappa)(f(\alpha))=a_{\alpha}
$$

holds by elementarity.

Of course, if a given Laver function works for many degrees of supercompactness then the joint Laver sequence derived above will work for those same degrees. In 
particular, if $\kappa$ is fully supercompact then this observation, combined with Laver's original construction, gives us a supercompactness joint Laver sequence of length $2^{\kappa}$.

Corollary 5. If $\kappa$ is supercompact then $\Delta_{\kappa, 2^{\kappa}}^{\mathrm{sc}}$ holds.

It should be pointed out that Laver's original argument only shows that a $\theta$ supercompactness Laver function exists for a cardinal $\kappa$ provided $\kappa$ is somewhere in the range of $2^{\theta^{<\kappa}}$-supercompact. Plain $\theta$-supercompactness does not suffice in the case $\theta=\kappa$ (that is, in the measurable case), since there are no Laver functions in Kunen's model $L[U]$ (as will follow from Proposition 13). It is currently unknown whether the hypothesis from Laver's proof can be reduced to just $\theta$ supercompactness in the case that $\kappa<\theta .^{2}$ We will not say much about this question in the present paper, and, since we are mainly interested in jointness phenomena, we will liberally assume that the large cardinal in question carries at least one Laver function.

Proposition 4 essentially shows that joint Laver sequences of maximal length exist automatically for cardinals with a high degree of supercompactness that carry at least one Laver function. Since we will be interested in comparing the strengths of the principles $\Delta_{\kappa, \lambda}$ for various $\lambda$, we will in the remainder of this section be mostly concerned with cardinals $\kappa$ which are not $2^{\kappa}$-supercompact (but are at least measurable), so as to avoid situations where a single Laver function gives rise to the longest possible joint Laver sequence.

3.1. Creating long joint Laver diamonds. We now show that the existence of $\theta$-supercompactness joint Laver sequences of maximal length does not require strength beyond $\theta$-supercompactness itself.

The following notion is due to Hamkins [10], although its original form, relating to strong compactness, dates back to Menas [19].

Definition 6. A $\theta$-supercompactness Menas function for a cardinal $\kappa$ is a function $f: \kappa \rightarrow \kappa$ such that there is a $\theta$-supercompactness embedding $j: V \rightarrow M$ with $\operatorname{cp}(j)=\kappa$ and $j(f)(\kappa)>\theta$.

A Menas function is a particularly weak form of a Laver function. If $\ell$ is a $\theta$ supercompactness Laver function for $\kappa$, then it is also a $\theta$-supercompactness Menas function, since we can pick the embedding $j$ to have $\ell$ guess $\theta+1$, for example. However, the advantage of Menas functions is that we can prove their existence from the optimal large cardinal hypothesis on $\kappa$, something which is unknown for Laver functions, as we mentioned in the preceding subsection.

Lemma 7. If $\kappa$ is $\theta$-supercompact, then $\kappa$ carries a $\theta$-supercompactness Menas function.

It is unclear who this lemma should be attributed to; we heard the following proof from Hamkins.

Proof. We consider the nontrivial case when $\kappa \leq \theta$. Define a function $f: \kappa \rightarrow \kappa$ by letting $f(\alpha)=0$ if $\alpha$ is $\kappa$-supercompact, and $f(\alpha)=2^{\lambda^{<\alpha}}$ where $\lambda<\kappa$ is least such that $\alpha$ is not $\lambda$-supercompact. It is simple to check that $f$ really maps into $\kappa$, since

\footnotetext{
${ }^{2}$ We might hope that recent work in providing a canonical inner model for finite levels of supercompactness will go some way towards answering this question.
} 
if $\alpha<\kappa$ is $\lambda$-supercompact for every $\lambda<\kappa$, then it is $\theta$-supercompact. We claim that this $f$ is a Menas function.

Let $j: V \rightarrow M$ be a $\theta$-supercompactness embedding with critical point $\kappa$ such that $\kappa$ is not $\theta$-supercompact in $M$. One can find such an embedding by either considering the Mitchell order on normal measures on $\mathcal{P}_{\kappa}(\theta)$ and taking the embedding corresponding to a minimal such measure, or by simply taking a $\theta$-supercompactness embedding $j$ for which $j(\kappa)$ is least among all such embeddings. ${ }^{3}$ Let us see that $j(f)(\kappa)>\theta$.

Since $\kappa$ is not $\theta$-supercompact in $M$ and $j(\kappa)>\theta$, we see that $j(f)(\kappa)=\left(2^{\lambda^{<\kappa}}\right)^{M}$, where $\lambda$ is the least such that $\kappa$ is not $\lambda$-supercompact in $M$. So suppose that $\left(2^{\lambda^{<\kappa}}\right)^{M} \leq \theta$. Since $M$ is closed under $\theta$-sequences, this means that $M$ computes $\mathcal{P}_{\kappa}(\lambda)$ and $\mathcal{P}\left(\mathcal{P}_{\kappa}(\lambda)\right)$ correctly and, in fact, contains every subset of $\mathcal{P}\left(\mathcal{P}_{\kappa}(\lambda)\right)$ from $V$. But $V$ has a normal measure on $\mathcal{P}_{\kappa}(\lambda)$, which is a subset just like that, and so $M$ must also have this normal measure. This means that $\kappa$ is in fact $\lambda$-supercompact in $M$, contradicting our earlier assumption.

Theorem 8. If $\kappa$ is $\theta$-supercompact, then there is a forcing extension in which $\Delta_{\kappa, 2^{\kappa}}^{\theta-\mathrm{sc}}$ holds.

It should be mentioned that the forcing we do in the course of the proof may collapse $2^{\kappa}$, and so the $\Delta_{\kappa, 2^{\kappa}}^{\theta-\mathrm{sc}}$ in the conclusion of the theorem should be read with the extension's version of $2^{\kappa}$.

Proof. Since the $\theta$-supercompactness of $\kappa$ implies its $\theta^{<\kappa}$-supercompactness (see, for example, [15, Proposition 22.11(b)]), we may assume that $\theta^{<\kappa}=\theta$. Furthermore, we assume that $2^{\theta}=\theta^{+}$, since this may be forced without adding subsets to $\mathcal{P}_{\kappa}(\theta)$ (which means that any measure on $\mathcal{P}_{\kappa}(\theta)$ remains a measure) or functions $\mathcal{P}_{\kappa}(\theta) \rightarrow \theta$ (which means that any normal measure remains normal), and so $\kappa$ will remain $\theta$-supercompact after this forcing. Fix a Menas function $f$ for $\kappa$ as in Lemma 7. Let $\mathbb{P}_{\kappa}$ be the length $\kappa$ Easton support iteration which forces with $\mathbb{Q}_{\gamma}=\operatorname{Add}\left(\gamma, 2^{\gamma}\right)$ at inaccessible closure points of $f$, meaning those inaccessible $\gamma$ for which $f[\gamma] \subseteq \gamma$. Finally, let $\mathbb{P}=\mathbb{P}_{\kappa} * \mathbb{Q}_{\kappa}$. Let $G * g \subseteq \mathbb{P}$ be generic; we will extract a joint Laver sequence from $g$.

If $g(\alpha)$ is the $\alpha$-th subset added by $g$, we view it as a sequence of bits. Using some coding scheme which admits end-of-code markers we can, given any $\xi<\kappa$, view the segment of $g(\alpha)$ between the $\xi$ th bit and the next marker as the Mostowski code of an element of $V_{\kappa}$. We then define $\ell_{\alpha}: \kappa \rightarrow V_{\kappa}$ as follows: given an inaccessible $\xi$, let $\ell_{\alpha}(\xi)$ be the set coded by $g(\alpha)$ at $\xi$; otherwise let $\ell_{\alpha}(\xi)=\emptyset$. We claim that $\left\langle\ell_{\alpha} ; \alpha<2^{\kappa}\right\rangle$ is a joint Laver sequence.

Let $\vec{a}=\left\langle a_{\alpha} ; \alpha<2^{\kappa}\right\rangle$ be a sequence of targets in $H_{\theta^{+}}^{V[G][g]}$. Let $j: V \rightarrow M$ be the ultrapower embedding by a normal measure on $\mathcal{P}_{\kappa}(\theta)$ which corresponds to $f$, meaning the one for which $j(f)(\kappa)>\theta$. We will lift this embedding through the forcing $\mathbb{P}$ in $V[G][g]$.

The argument splits into two cases, depending on the size of $\theta$. We deal first with the easier case when $\theta \geq 2^{\kappa}$. In this case the poset $j\left(\mathbb{P}_{\kappa}\right)$ factors as $j\left(\mathbb{P}_{\kappa}\right)=$ $\mathbb{P}_{\kappa} * \mathbb{Q}_{\kappa} * \mathbb{P}_{\text {tail }}$. Since $j(f)(\kappa)>\theta$, the next stage of forcing in $j\left(\mathbb{P}_{\kappa}\right)$ above $\kappa$

\footnotetext{
${ }^{3}$ In either case, the key observation is that, if $\mu$ and $\nu$ are normal measures on $\mathcal{P}_{\kappa}(\theta)$ and $\mu$ appears in the ultrapower by $\nu$, then $j_{\mu}(\kappa)<j_{\nu}(\kappa)$, where $j_{\mu}$ and $j_{\nu}$ are the corresponding embeddings.
} 
occurs after $\theta$, so $\mathbb{P}_{\text {tail }}$ is $\leq \theta$-closed in $M[G][g]$ and has size $j(\kappa)$ there. It follows that $\mathbb{P}_{\text {tail }}$ has $j\left(2^{\kappa}\right)$ many subsets in $M[G][g]$. Since $M$ was an ultrapower by a normal measure on $\mathcal{P}_{\kappa}(\theta)$, the ordinal $j\left(2^{\kappa}\right)$ has size at most $\left(2^{\kappa}\right)^{\theta}=\theta^{+}$in $V[G][g]$ (as every smaller ordinal is represented by a function $\mathcal{P}_{\kappa}(\theta) \rightarrow 2^{\kappa}$ ). Therefore, $V[G][g]$ sees that there are only $\theta^{+}$many dense subsets of $\mathbb{P}_{\text {tail }}$ in $M[G][g]$. These can be lined up and met one at a time, using both that $M[G][g]$ is closed under $\theta$-sequences in $V[G][g]$ and that $\mathbb{P}_{\text {tail }}$ is a $\leq \theta$-closed poset in $M[G][g]$. This process produces in $V[G][g]$ an $M[G][g]$-generic $G_{\text {tail }} \subseteq \mathbb{P}_{\text {tail }}$ and allows us to lift $j$ to $j: V[G] \rightarrow M[j(G)]$, where $j(G)=G * g * G_{\text {tail }}$.

Since $M[j(G)]$ is still an ultrapower and thus closed under $\theta$-sequences in $V[G][g]$, we get $j[g] \in M[j(G)]$. Since $j\left(\mathbb{Q}_{\kappa}\right)$ is $\leq \theta$-directed closed in $M[j(G)]$ it has $q=\bigcup j[g]$ as a condition. This $q$ is a partial function on the domain $j\left(2^{\kappa}\right) \times \kappa$. Since $M[j(G)]$ has both the sequence of targets $\vec{a}$ and $j\left\lceil 2^{\kappa}\right.$, we can further extend $q$ to $q^{*} \in M[j(G)]$ by coding $a_{\alpha}$ into the bit-sequence of $q(j(\alpha))$ at $\kappa$ for each $\alpha<2^{\kappa}$. We again diagonalize against the $\left|2^{2^{j(\kappa)}}\right| \leq \theta^{+}$many dense subsets of $j\left(\mathbb{Q}_{\kappa}\right)$ in $M[j(G)]$ below the master condition $q^{*}$ to get a $M[j(G)]$-generic $g^{*} \subseteq j\left(\mathbb{Q}_{\kappa}\right)$ and lift $j$ to $j: V[G][g] \rightarrow M[j(G)]\left[g^{*}\right]$. Finally, observe that we have arranged the construction of $g^{*}=j(g)$ in such a way that $g^{*}(j(\alpha))$ codes $a_{\alpha}$ at $\kappa$ for all $\alpha<2^{\kappa}$ and, by definition, this implies that $j\left(\ell_{\alpha}\right)(\kappa)=a_{\alpha}$ for all $\alpha<2^{\kappa}$. Thus we indeed have a joint Laver sequence for $\kappa$ of length $2^{\kappa}$ in $V[G][g]$.

It remains for us to consider the second case, when $\kappa \leq \theta<2^{\kappa}$. In this situation our assumptions on $\theta$ imply that $2^{\kappa}=\theta^{+}$. The poset $j\left(\mathbb{P}_{\kappa}\right)$ factors as $j\left(\mathbb{P}_{\kappa}\right)=$ $\mathbb{P}_{\kappa} * \widetilde{\mathbb{Q}}_{\kappa} * \mathbb{P}_{\text {tail }}$, where $\widetilde{\mathbb{Q}}_{\kappa}=\operatorname{Add}\left(\kappa,\left(2^{\kappa}\right)^{M[G]}\right)$. Since $V[G]$ and $M[G]$ agree on $\mathcal{P}(\kappa)$, the ordinal $\left(2^{\kappa}\right)^{M[G]}$ has size $2^{\kappa}$ in $V[G]$, so $\widetilde{\mathbb{Q}}_{\kappa}$ is isomorphic, but not necessarily equal, to $\mathbb{Q}_{\kappa}$. Nevertheless, the same argument as before allows us to lift $j$ to $j: V[G] \rightarrow M[j(G)]$ where $j(G)=G * \widetilde{g} * G_{\text {tail }}$ and $\widetilde{g}$ is the isomorphic image of $g$.

We seem to hit a snag with the final lift through the forcing $\mathbb{Q}_{\kappa}$, which has size $2^{\kappa}$ and thus resists the usual approach of lifting via a master condition, since this condition would simply be too big for the amount of closure we have. We salvage the argument by using a technique, originally due to Magidor [18], sometimes known as the "master filter argument".

The forcing $j\left(\mathbb{Q}_{\kappa}\right)=\operatorname{Add}\left(j(\kappa), 2^{j(\kappa)}\right)^{M[j(G)]}$ has size $2^{j(\kappa)}$ and is $\leq \theta$-directed closed and $j(\kappa)^{+}$-cc in $M[j(G)]$. Since $M[j(G)]$ is still an ultrapower, $\left|2^{j(\kappa)}\right| \leq \theta^{+}=$ $2^{\kappa}$ and so $M[j(G)]$ has at most $2^{\kappa}$ many maximal antichains of $j\left(\mathbb{Q}_{\kappa}\right)$, counted in $V[G][g]$. Let these be given in the sequence $\left\langle Z_{\alpha} ; \alpha<2^{\kappa}\right\rangle$. Since each $Z_{\alpha}$ has size at most $j(\kappa)$, it is in fact contained in some bounded part of the poset $j\left(\mathbb{Q}_{\kappa}\right)$. Furthermore (and crucially), since $j$ is an ultrapower by a measure on $\mathcal{P}_{\kappa}(\theta)$, it is continuous at $2^{\kappa}=\theta^{+}$and so there is for each $\alpha$ a $\beta_{\alpha}<2^{\kappa}$ such that $Z_{\alpha} \subseteq$ $\operatorname{Add}\left(j(\kappa), j\left(\beta_{\alpha}\right)\right)$. In particular, each $Z_{\alpha}$ is a maximal antichain in $\operatorname{Add}\left(j(\kappa), j\left(\beta_{\alpha}\right)\right)$. We will now construct in $V[G][g]$ a descending sequence of conditions, deciding more and more of the antichains $Z_{\alpha}$, which will generate a filter, the "master filter", that will allow us to lift $j$ to $V[G][g]$ and also (lest we forget) witness the joint guessing property. We begin by defining the first condition $q_{0}$. Consider the generic $g$ up to $\beta_{0}$. This piece has size $\theta$ and so $\bigcup j\left[g\left\lceil\beta_{0}\right]\right.$ is a condition in $j\left(\mathbb{Q}_{\kappa}\right)\left\lceil j\left(\beta_{0}\right)\right.$. Let $q_{0}^{\prime}$ be the extension of $\bigcup j\left[g\left\lceil\beta_{0}\right]\right.$ which codes the target $a_{\alpha}$ at $\kappa$ in $q_{0}^{\prime}(j(\alpha))$ for each $\alpha<\beta_{0}$. This is still a condition in $j\left(\mathbb{Q}_{\kappa}\right)\left\lceil j\left(\beta_{0}\right)\right.$ and we can finally let $q_{0}$ be any extension of $q_{0}^{\prime}$ in this poset which decides the maximal antichain $Z_{0}$. Note that $q_{0}$ is compatible with every condition in $j[g]$, since we extended the partial 
master condition $\bigcup j\left[g\left\lceil\beta_{0}\right]\right.$ and made no commitments outside $j\left(\mathbb{Q}_{\kappa}\right)\left\lceil j\left(\beta_{0}\right)\right.$. We continue in this way recursively, constructing a descending sequence of conditions $q_{\alpha}$ for $\alpha<\theta^{+}$, using the closure of $j\left(\mathbb{Q}_{\kappa}\right)$ and $M[j(G)]$ to pass through limit stages. Now consider the filter $g^{*}$ generated by the conditions $q_{\alpha}$. It is $M[j(G)]$-generic by construction and also extends (or can easily be made to extend) $j[g]$. We can thus lift $j$ to $j: V[G][g] \rightarrow M[j(G)]\left[g^{*}\right]$ and, since $\mathbb{P}$ is $\theta^{+}$-cc and both $G_{\text {tail }}$ and $g^{*}$ were constructed in $V[G][g]$, the model $M[j(G)]\left[g^{*}\right]$ is closed under $\theta$-sequences which shows that $\kappa$ remains $\theta$-supercompact in $V[G][g]$. Finally, as in the previous case, $g^{*}$ was constructed in such a way that $j\left(\ell_{\alpha}\right)(\kappa)=a_{\alpha}$ for all $\alpha<2^{\kappa}$, verifying that these functions really do form a joint Laver sequence for $\kappa$.

As a special case of Theorem 8 we can deduce the corresponding result for measurable cardinals.

Corollary 9. If $\kappa$ is measurable, then there is a forcing extension in which there is a joint Laver sequence for $\kappa$ of length $2^{\kappa}$.

It follows from the results of Hamkins [12] that the forcing $\mathbb{P}$ from Theorem 8 does not create any measurable or (partially) supercompact cardinals below $\kappa$, since it admits a very low gap. We could therefore have started with the least large cardinal $\kappa$ of interest and preserved its minimality throughout the construction.

Corollary 10. If $\kappa$ is the least $\theta$-supercompact cardinal, then there is a forcing extension where $\kappa$ remains the least $\theta$-supercompact cardinal and $\Delta_{\kappa, 2^{\kappa}}^{\theta-\mathrm{sc}}$ holds.

It is perhaps interesting to observe the peculiar arrangement of cardinal arithmetic in the model produced in the above proof. We have $2^{\theta}=\theta^{+}$and, if $\theta \leq 2^{\kappa}$, also $2^{\kappa}=\theta^{+}$. In particular, we never produced a $\theta$-supercompactness joint Laver sequence of length greater than $\theta^{+}$(assuming here, of course, that $\theta=\theta^{<\kappa}$ is the optimal degree of supercompactness). One has to wonder whether this is significant. Certainly the existence of long joint Laver sequences does not imply much about cardinal arithmetic, since, for example, if $\kappa$ is indestructibly supercompact, we can manipulate the value of $2^{\kappa}$ freely, while maintaining the existence of a supercompactness joint Laver sequence of length $2^{\kappa}$. On the other hand, even in the case of measurable $\kappa$, the consistency strength of $2^{\kappa}>\kappa^{+}$is known to exceed that of $\kappa$ being measurable. The following question is therefore natural:

Question 11. If $\kappa$ is $\theta$-supercompact and $2^{\kappa}>\theta^{+}$, is there a forcing extension preserving these facts in which there is a joint Laver sequence for $\kappa$ of length $2^{\kappa}$ ?

We next show that the existence of joint Laver sequences is preserved under mild forcing. This will be useful later when we separate the existence of these sequences based on their lengths.

Lemma 12. Let $\kappa$ be $\theta$-supercompact (with $\kappa \leq \theta$ ), $\lambda$ a cardinal, and assume $\Delta_{\kappa, \lambda}^{\theta \text {-sc }}$ holds. Suppose $\mathbb{P}$ is a poset such that either

(1) $\lambda \geq \theta^{<\kappa}$ and $\mathbb{P}$ is $\leq \lambda$-distributive, or

(2) $|\mathbb{P}| \leq \kappa$ and, for any $\lambda$-sequence of targets, some embedding associated to the joint Laver sequence and these targets lifts through $\mathbb{P}$.

Then forcing with $\mathbb{P}$ preserves $\star_{\kappa, \lambda}^{\theta \text {-sc }}$.

If $|\mathbb{P}| \leq \kappa$, it is very often the case that every $\theta$-supercompactness embedding with critical point $\kappa$ lifts through $\mathbb{P}$, so the hypothesis in item (2) is easily satisfied. 
Furthermore, while the restriction $|\mathbb{P}| \leq \kappa$ in (2) is necessary for full generality, it can in fact be relaxed to $|\mathbb{P}| \leq \theta$ for a large class of forcings.

Proof. Under the hypotheses of (1) every ultrapower embedding by a measure on $\mathcal{P}_{\kappa}(\theta)$ lifts to the extension by $\mathbb{P}$ (see, for example, [6, Proposition 15.1]) and no elements of $H_{\theta^{+}}$or $\lambda$-sequences of these are added, so any ground model joint Laver sequence of length $\lambda$ remains such in the extension.

Now suppose that the hypotheses of (2) hold, let $\left\langle\ell_{\alpha} ; \alpha<\lambda\right\rangle$ be a joint Laver sequence for $\kappa$ and let $G \subseteq \mathbb{P}$ be generic. We may also assume that the underlying set of $\mathbb{P}$ is a subset of $\kappa$. Define functions $\ell_{\alpha}^{\prime}: \kappa \rightarrow V_{\kappa}[G]$ by $\ell_{\alpha}^{\prime}(\xi)=\ell_{\alpha}(\xi)^{G \cap \xi}$ if this makes sense and $\ell_{\alpha}^{\prime}(\xi)=\emptyset$ otherwise. We claim that $\left\langle\ell_{\alpha}^{\prime} ; \alpha<\lambda\right\rangle$ is a joint Laver sequence in $V[G]$.

Let $\vec{a}$ be a $\lambda$-sequence of targets in $H_{\theta^{+}}^{V[G]}$, and let $\dot{\vec{a}}$ be a name for $\vec{a}$. We can use this name and the fullness property to derive names $\dot{a}_{\alpha}$ for the targets $a_{\alpha}$, uniformly in $\alpha<\lambda$, which means that we find the entire sequence $\left\langle\dot{a}_{\alpha} ; \alpha<\lambda\right\rangle$ in $V$. Since each $\dot{a}_{\alpha}$ names an element of $H_{\theta^{+}}^{V[G]}$, and because $\mathbb{P}$ is small (and therefore $\theta^{+}$-cc), we can find a nice name $\sigma_{\alpha} \in H_{\theta^{+}}$for each $\dot{a}_{\alpha}$, and the sequence of these nice names is in $V$ as well. Now let $j: V \rightarrow M$ be a $\theta$-supercompactness embedding with critical point $\kappa$ which lifts through $\mathbb{P}$ and which satisfies $j\left(\ell_{\alpha}\right)(\kappa)=\sigma_{\alpha}$ for each $\alpha$. It then follows that $j\left(\ell_{\alpha}^{\prime}\right)(\kappa)=a_{\alpha}$ in $V[G]$, verifying the joint Laver diamond property there.

3.2. Separating joint Laver diamonds by length. We next aim to show that it is consistent that there is a measurable Laver function for $\kappa$ but no joint Laver sequences of length $\kappa^{+}$. The following proposition expresses the key observation for our solution, connecting the question to the number of normal measures problem.

Proposition 13. If there is a $\theta$-supercompactness joint Laver sequence for $\kappa$ of length $\lambda$, then there are at least $2^{\theta \cdot \lambda}$ many normal measures on $\mathcal{P}_{\kappa}(\theta)$.

Proof. The point is that any $\lambda$-sequence of targets in $H_{\theta^{+}}$can be guessed via the embedding arising from a normal measure on $\mathcal{P}_{\kappa}(\theta)$ (see the discussion at the start of Section 3) and any such measure realizes a single $\lambda$-sequence via the fixed joint Laver sequence. But there are $2^{\theta \cdot \lambda}$ many sequences of targets, and thus there must be at least this many measures.

Theorem 14. If $\kappa$ is measurable, then there is a forcing extension in which there is a Laver function for $\kappa$ but no joint Laver sequence of length $\kappa^{+}$.

Proof. After forcing as in the proof of Theorem 8, if necessary, we may assume that $\kappa$ has a Laver function. A result of Apter, Cummings, and Hamkins [1] then shows that $\kappa$ still carries a Laver function in the extension by $\mathbb{P}=\operatorname{Add}(\omega, 1) * \operatorname{Coll}\left(\kappa^{+}, 2^{2^{\kappa}}\right)$, but only carries $\kappa^{+}$many normal measures there. Proposition 13 now implies that there cannot be a joint Laver sequence of length $\kappa^{+}$in the extension.

We can push this result a bit further to get a separation between any two desired lengths of joint Laver sequences. To state the sharpest result we need to introduce a new notion.

Definition 15. Let $\kappa$ be a large cardinal supporting a notion of Laver diamond and $\lambda$ a cardinal. We say that a sequence $\vec{\ell}=\left\langle\ell_{\alpha} ; \alpha<\lambda\right\rangle$ is an almost-joint Laver 
sequence for $\kappa$ if $\vec{\ell} \uparrow \gamma$ is a joint Laver sequence for $\kappa$ for any $\gamma<\lambda$. We say that $\Delta_{\kappa,<\lambda}$ holds if there is an almost-joint Laver sequence of length $\lambda$.

Theorem 16. Suppose $\kappa$ is measurable and let $\lambda$ be a regular cardinal satisfying $\kappa<\lambda \leq 2^{\kappa}$. If $\triangle_{\kappa,<\lambda}^{\text {meas }}$ holds then there is a forcing extension preserving this in which $\AA_{\kappa, \lambda}^{\text {meas }}$ fails.

Proof. We imitate the proof of Theorem 14 but force instead with $\mathbb{P}=\operatorname{Add}(\omega, 1) *$ $\operatorname{Coll}\left(\lambda, 2^{2^{\kappa}}\right)$. The analysis based on [1] now shows that the final extension has at most $\lambda$ many normal measures on $\kappa$ and thus there can be no joint Laver sequences of length $\lambda$ there by Proposition 13. That $\Delta_{\kappa,<\lambda}^{\text {meas }}$ still holds follows from (the proof of) Lemma 12: part (2) implies that, by guessing names, the $\Delta_{\kappa,<\lambda}^{\text {meas }}$-sequence from the ground model gives rise to one in the intermediate Cohen extension. Part (1) then shows that each of the initial segments of this sequence remains a joint Laver sequence in the final extension.

We can also extend these results to $\theta$-supercompact cardinals without too much effort.

Theorem 17. If $\kappa$ is $\theta$-supercompact, $\theta$ is regular, and $\theta^{<\kappa}=\theta$, then there is a

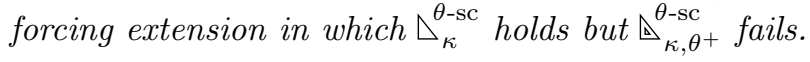

Of course, the theorem is only interesting when $\kappa \leq \theta<2^{\kappa}$, in which case the given separation is best possible in view of Proposition 4 .

Proof. We may assume by prior forcing, as in Theorem 8, that we have a Laver function for $\kappa$. We now force with $\mathbb{P}=\operatorname{Add}(\omega, 1) * \operatorname{Coll}\left(\theta^{+}, 2^{2^{\theta}}\right)$ to get an extension $V[g][G]$. By the results of $[1]$, the extension $V[g][G]$ has at most $\theta^{+}$many normal measures on $\mathcal{P}_{\kappa}(\theta)$ and therefore there are no joint Laver sequences for $\kappa$ of length $\theta^{+}$there by Proposition 13. It remains to see that there is a Laver function in $V[g][G]$. Let $\ell$ be a Laver function in $V$ and define $\ell^{\prime} \in V[g][G]$ by $\ell^{\prime}(\xi)=\ell(\xi)^{g}$ if $\ell(\xi)$ is an $\operatorname{Add}(\omega, 1)$-name and $\ell^{\prime}(\xi)=\emptyset$ otherwise. For a given $a \in H_{\theta^{+}}^{V[g][G]}=$ $H_{\theta^{+}}^{V[g]}$ we can select an $\operatorname{Add}(\omega, 1)$-name $\dot{a} \in H_{\kappa^{+}}^{V}$ and find a $\theta$-supercompactness embedding $j: V \rightarrow M$ such that $j(\ell)(\kappa)=\dot{a}$. The embedding $j$ lifts to $j: V[g][G] \rightarrow$ $M[g][j(G)]$ since the Cohen forcing was small and the collapse forcing was $\leq \theta$ closed. But then clearly $j\left(\ell^{\prime}\right)(\kappa)=\dot{a}^{g}=a$, so $\ell^{\prime}$ is a Laver function.

Theorem 18. Suppose $\kappa$ is $\theta$-supercompact and let $\lambda$ be a regular cardinal satisfying

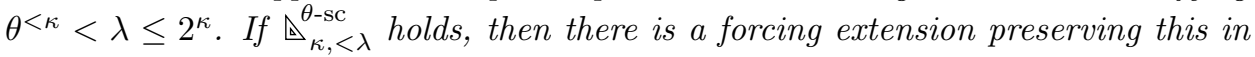
which ${\Delta_{\kappa, \lambda}^{\theta-s c}}_{\text {fails. }}$

Proof. The relevant forcing is $\operatorname{Add}(\omega, 1) * \operatorname{Coll}\left(\lambda, 2^{2^{\theta^{<\kappa}}}\right)$. Essentially the argument from Theorem 16 then finishes the proof.

Just as with joint Laver sequences, there is an upper bound on the length of an almost-joint Laver sequence.

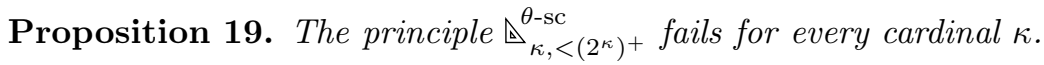

Proof. Any potential $\Delta_{\kappa,<\left(2^{\kappa}\right)^{+}}^{\theta-s e q u e n c e ~ m u s t ~ n e c e s s a r i l y ~ h a v e ~ t h e ~ s a m e ~ f u n c t i o n ~}$ appear on at least two coordinates. But then any initial segment of this sequence 
containing both of those coordinates cannot be joint, since it cannot guess distinct targets on those coordinates.

A question remains about the principles $\unlhd_{\kappa,<\lambda}$, whether they are genuinely new or whether they reduce to other principles.

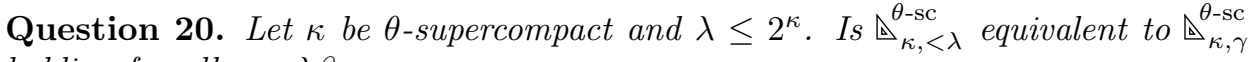
holding for all $\gamma<\lambda$ ?

An almost-joint Laver sequence definitely gives instances of joint Laver diamonds of each particular length $\gamma$. The reverse implication is especially interesting in the case when $\lambda=\mu^{+}$is a successor cardinal. This is because simply rearranging the functions in a joint Laver sequence of length $\mu$ gives joint Laver sequences of any length shorter than $\mu^{+}$. The question is thus asking whether $\Delta_{\kappa, \mu}$ suffices for $\triangle_{\kappa,<\mu^{+}}$.

An annoying feature of the models produced in the preceding theorems is that in all of them the least $\lambda$ for which $\Delta_{\kappa, \lambda}$ fails is $\lambda=2^{\kappa}$. One has to wonder whether this is significant.

In particular, we would like an answer to the following question: is it relatively consistent that there is a $\theta$-supercompact cardinal $\kappa$, for some $\theta$, such that $\triangle_{\kappa}^{\theta \text {-sc }}$ holds and $\AA_{\kappa, \lambda}^{\theta \text {-sc }}$ fails for some $\lambda<2^{\kappa}$ ?

To satisfy the listed conditions, GCH must fail at $\kappa$ (since we must have $\kappa<$ $\lambda<2^{\kappa}$ by Proposition 4). We can therefore expect that achieving the situation described in the question will require some additional consistency strength.

In the case of a measurable $\kappa$ the answer to this question is positive: we will show in Theorem 22 that, starting from sufficient large cardinal hypotheses, we can produce a model where $\kappa$ is measurable and has a measurable Laver function but no joint Laver sequences of length $\kappa^{+}<2^{\kappa}$. The proof relies on an argument due to Friedman and Magidor [8] which facilitates the simultaneous control of the number of measures at $\kappa$ and the value of the continuum function at $\kappa$ and $\kappa^{+}$.

Let us briefly give a general setup for the argument of [8] that will allow us to carry out our intended modifications without repeating too much of the work done there.

We first recall the higher Sacks forcing, originally due to Kanamori [14]. Let $\gamma$ be an inaccessible cardinal. A condition in the poset $\operatorname{Sacks}(\gamma)$ is a $<\gamma$-closed subtree $T$ of ${ }^{<\gamma} 2$ of height $\gamma$, such that there is a club $C_{T} \subseteq \gamma$ such that a node $t \in T$ is a splitting node in $T$ if and only if $|t| \in C_{T}$. Stronger conditions are given by subtrees. A generic filter for $\operatorname{Sacks}(\gamma)$ determines (and is, in turn, determined by) a single branch through ${ }^{<\gamma} 2$, a new subset of $\gamma$ in the extension. The forcing is $<\gamma$-closed and satisfies a form of fusion, which means that it preserves $\gamma^{+}$as well.

As a slight generalization, one might consider bushier trees instead of just binary trees as conditions. Of particular interest will be the version in which conditions are $<\gamma$-closed subtrees of ${ }^{<\gamma} \gamma$ of height $\gamma$, where each splitting node of height $\delta$ (and there is again a club of these heights of splitting nodes) splits into $\delta^{++}$many immediate successors. Call this version of the forcing $\operatorname{Sacks}_{\mathrm{id}^{++}}(\gamma)$.

Fix a cardinal $\kappa$ and suppose GCH holds up to and including $\kappa$. Furthermore suppose that $\kappa$ is the critical point of an elementary embedding $j: V \rightarrow M$ satisfying the following properties:

- $j$ is an extender embedding, meaning that every element of $M$ has the form $j(f)(\alpha)$ for some function $f$ defined on $\kappa$ and some $\alpha<j(\kappa)$. 
- $\left(\kappa^{++}\right)^{M}=\kappa^{++}$.

- There is a function $f: \kappa \rightarrow V$, such that $j(f)(\kappa)$ is, in $V$ (and therefore also in $M$ ), a sequence of $\kappa^{++}$many disjoint stationary subsets of $\kappa^{++} \cap \operatorname{Cof}_{\kappa^{+}}$.

Given this arrangement, Friedman and Magidor define a forcing iteration $\mathbb{P}$ of length $\kappa+1$ (with nonstationary support) which forces at each inaccessible stage $\gamma \leq \kappa$ with $\operatorname{Sacks}^{*}\left(\gamma, \gamma^{++}\right) * \operatorname{Sacks}_{\mathrm{id}^{++}}(\gamma) * \operatorname{Code}(\gamma)$. Here $\operatorname{Sacks}^{*}\left(\gamma, \gamma^{++}\right)$is a large product of versions of $\operatorname{Sacks}(\gamma)$ where the splitting levels are restricted to the singular elements of a club and $\operatorname{Code}(\gamma)$ is a certain $\leq \gamma$-distributive notion of forcing coding information about the stage $\gamma$ generics into the stationary sets given by $f(\gamma)$.

Let $G \subseteq \mathbb{P}$ be generic. We shall use $\mathbb{P}_{<\kappa}$ to denote the initial segment of the iteration $\mathbb{P}$ up to stage $\kappa$, and $G_{<\kappa}$ will be the corresponding restriction of the generic $G$. In the interest of avoiding repeating the analysis of the forcing notion given in [8], we list some of the properties of the extension $V[G]$ that we will use (but see [8] for proofs):

(1) $\mathbb{P}$ preserves cardinals and cofinalities, and increases the values of the continuum function by at most two cardinal steps. In particular, any inaccessible cardinals of $V$ remain such in $V[G]$.

(2) We have $2^{\kappa}=\kappa^{++}$in $V[G]$.

(3) $\mathbb{P}$ has the $\kappa$-Sacks property: for any function $f: \kappa \rightarrow \operatorname{Ord}$ in $V[G]$ there is a function $h \in V$ such that $f(\alpha) \in h(\alpha)$ for all $\alpha$ and $|h(\alpha)| \leq \alpha^{++}$.

(4) The generic $G$ is self-encoding in a strong way: in $V[G]$ there is a unique $M$-generic for $j(\mathbb{P})_{<j(\kappa)}$ extending $j\left[G_{<\kappa}\right]$.

(5) If $S_{\kappa}$ is the generic added by $\operatorname{Sacks}_{\mathrm{id}^{++}}(\kappa)$ within $\mathbb{P}$, then $\bigcap j\left[S_{\kappa}\right]$ is a tuning fork: the union of $\kappa^{++}$many branches, all of which split off exactly at level $\kappa$.

(6) In $V[G]$ there are exactly $\kappa^{++}$many $M$-generics for $j(\mathbb{P})$ extending $j[G]$, corresponding to the $\kappa^{++}$many branches in $\bigcap j\left[S_{\kappa}\right]$. In particular, there are exactly $\kappa^{++}$many lifts $j_{\alpha}$ of $j$ to $V[G]$, distinguished by $j_{\alpha}\left(S_{\kappa}\right)(\kappa)=\alpha$ for $\alpha<\kappa^{++}$.

Proposition 21. In the above setup, the iteration $\mathbb{P}$ adds a measurable Laver function for $\kappa$.

Proof. Let $G \subseteq \mathbb{P}$ be generic. As we stated in item (6), for any $\alpha<\kappa^{++}$there is a lift $j_{\alpha}: V[G] \rightarrow M\left[j_{\alpha}(G)\right]$ of $j$ such that $j_{\alpha}\left(S_{\kappa}\right)(\kappa)=\alpha$, where $S_{\kappa}$ is the Sacks subset of $\kappa$ added by the $\kappa$ th stage of $G$. This shows that $\bar{\ell}(\gamma)=S_{\kappa}(\gamma)$ is a $\kappa^{++}$-guessing measurable Laver function for $\kappa$.

Note that all of the subsets of $\kappa$ in $M\left[j_{\alpha}(G)\right]$ (and $V[G]$ ) appear already in $M[G]$; this is because the part of $j(\mathbb{P})$ above stage $\kappa$ is forced to be $\leq \kappa$-distributive. Let $\vec{e}=\left\langle e_{\alpha} ; \alpha<\kappa^{++}\right\rangle$be an enumeration of $H_{\kappa^{+}}^{M[G]}$ in $M[G]$ and let $\dot{e} \in M$ be a name for $\vec{e}$. We can write $\dot{e}=j(F)(\kappa)$ for some function $F$, defined on $\kappa$. Now define a function $\ell: \kappa \rightarrow V_{\kappa}$ in $V[G]$ by $\ell(\gamma)=\left(F(\gamma)^{G}\right)(\bar{\ell}(\gamma))$. This is, in fact, our desired Laver function; given an arbitrary element of $H_{\kappa^{+}}^{V[G]}$, we can find it in the enumeration $\vec{e}$. If $\alpha$ is its index, then

$$
j_{\alpha}(\ell)(\kappa)=\left(j_{\alpha}(F)(\kappa)^{j_{\alpha}(G)}\right)\left(j_{\alpha}(\bar{\ell})(\kappa)\right)=\left(j(F)(\kappa)^{j_{\alpha}(G)}\right)(\alpha)=\vec{e}(\alpha)=e_{\alpha} .
$$

Theorem 22. Suppose $\kappa$ is $(\kappa+2)$-strong and assume that $V=L[\vec{E}]$ is the minimal extender model witnessing this. Then there is a forcing extension in which $2^{\kappa}=$ 
$\kappa^{++}$, the cardinal $\kappa$ remains measurable, $\kappa$ carries a measurable Laver function, and there are no measurable joint Laver sequences for $\kappa$ of length $\kappa^{+}$.

Proof. Let $j: V \rightarrow M$ be the ultrapower embedding by the top extender of $\vec{E}$, the unique extender witnessing the $(\kappa+2)$-strongness of $\kappa$. In particular, every element of $M$ has the form $j(f)(\alpha)$ for some $\alpha<j(\kappa)$, and $M$ computes $\kappa^{++}$correctly. Furthermore, $V$ has a canonical $\nabla_{\kappa^{++}}\left(\mathrm{Cof}_{\kappa^{+}}\right)$-sequence, which is definable without parameters over $H_{\kappa^{++}}$via the standard condensation argument. Since $H_{\kappa^{++}} \in M$, this same sequence is also in $M$ and is of the form $j(\bar{f})(\kappa)$ for some function $\bar{f}$, since it is definable in $M$ just from the parameter $\kappa$. By having this diamond sequence guess the singletons $\{\xi\}$ for $\xi<\kappa^{++}$, we obtain a sequence of $\kappa^{++}$many disjoint stationary subsets of $\kappa^{++} \cap \operatorname{Cof}_{\kappa^{+}}$, and this sequence itself has the form $j(f)(\kappa)$ for some function $f$. We are therefore in a situation where the definition of the Friedman-Magidor iteration we described above makes sense. But first, we shall carry out some preliminary forcing.

Let $g \subseteq \operatorname{Add}\left(\kappa^{+}, \kappa^{+3}\right)$ be generic. Since this Cohen poset is $\leq \kappa$-distributive, the embedding $j$ lifts (uniquely) to an embedding $j: V[g] \rightarrow M[j(g)]{ }^{4}$ Let us examine the lifted embedding $j$. It is still an extender embedding. Additionally, since GCH holds in $V$, the forcing $\operatorname{Add}\left(\kappa^{+}, \kappa^{+3}\right)$ preserves cardinals, cofinalities, and stationary subsets of $\kappa^{++}$. Together, this means that $M[j(g)]$ computes $\kappa^{++}$correctly, and the stationary sets given by the sequence $j(f)(\kappa)$ above remain stationary. Therefore we may still define the Friedman-Magidor iteration $\mathbb{P}$ over $V[g]$.

Let $G \subseteq \mathbb{P}$ be generic over $V[g]$. We claim that $V[g][G]$ is the model we want. We have $2^{\kappa}=\kappa^{++}$in the extension, by item (2) of our list, and Proposition 21 implies that $\kappa$ is measurable in $V[g][G]$ and $\triangle_{\kappa}^{\text {meas }}$ holds there. So it remains for us to see that $\Delta_{\kappa, \kappa^{+}}^{\text {meas }}$ fails. By Proposition 13 it suffices to show that $\kappa$ does not carry $2^{\kappa^{+}}=\kappa^{+3}$ many normal measures in $V[g][G]$.

Let $U^{*} \in V[g][G]$ be a normal measure on $\kappa$ and let $j^{*}: V[g][G] \rightarrow N\left[j^{*}(g)\right]\left[j^{*}(G)\right]$ be its associated ultrapower embedding. This embedding restricts to $j^{*} \mid V: V \rightarrow N$. Since $V$ is the core model from the point of view of $V[g][G]$, the embedding $j^{*} \mid V$ arises as the ultrapower map associated to a normal iteration of extenders on the sequence $\vec{E}$ (see [22, Section 7.4] for more details).

We first claim that the first extender applied in this iteration is the top extender of $\vec{E}$. Let us write $j^{*}\left\lceil V=j_{1} \circ j_{0}\right.$, where $j_{0}: V \rightarrow N_{0}$ results from the first applied extender. Clearly $j_{0}$ has critical point $\kappa$. Now suppose that $j_{0}(\kappa)<\kappa^{++}$. Of course, $j_{0}(\kappa)$ is inaccessible in $N_{0}$ and, since $N$ is an inner model of $N_{0}$, also in $N$. But $j_{0}(\kappa)$ is not inaccessible in $N\left[j^{*}(g)\right]\left[j^{*}(G)\right]$, since $2^{\kappa}=\kappa^{++}$there. This is a contradiction, since passing from $N$ to $N\left[j^{*}(g)\right]\left[j^{*}(G)\right]$ preserves inaccessibility, by item (1) of our list.

It follows that we must have $j_{0}(\kappa) \geq \kappa^{++}$. We will argue that the extender $E$ applied to get $j_{0}$ witnesses the $(\kappa+2)$-strongness of $\kappa$, so it must be the top extender of $\vec{E}$. Using a suitable indexing of $\vec{E}$, the extender $E$ has index $\left(j_{0}(\kappa)^{+}\right)^{N_{0}}>\kappa^{++}$, and the coherence of the extender sequence implies that the sequences in $V$ and in $N_{0}$ agree up to $\kappa^{++}$. By the acceptability of these extender models it now follows that $H_{\kappa^{++}}^{V}=H_{\kappa^{++}}^{N_{0}}$ or, equivalently, $V_{\kappa+2} \in N_{0}$. This means that $j_{0}$ is the

\footnotetext{
${ }^{4}$ The lifted embedding will not be a $(\kappa+2)$-strongness embedding and, in fact, $\kappa$ is no longer $(\kappa+2)$-strong in $V[g]$. Nevertheless, the residue of strongness will suffice for our argument.
} 
$(\kappa+2)$-strongness ultrapower of $V$, and is equal to the embedding $j$ we started with.

Finally, we claim that the iteration giving rise to $j^{*} \uparrow V$ ends after one step, meaning that $j^{*} \mid V=j_{0}=j$. Suppose to the contrary that $j_{1}$ is nontrivial. By the normality of the iteration, the critical point of $j_{1}$ must be some $\lambda>\kappa$. We can find a function $h \in V[g][G]$, defined on $\kappa$, such that $j^{*}(h)(\kappa)=\lambda$, since $j^{*}$ is given by an ultrapower of $V[g][G]$ by a normal measure on $\kappa$. By the $\kappa$-Sacks property of $\mathbb{P}$ (see item (3)) we can cover the function $h$ by a function $\bar{h} \in V[g]$; in fact, since the forcing to add $g$ was $\leq \kappa$-closed, we have $\bar{h} \in V$. Now

$$
\lambda=j^{*}(h)(\kappa) \in j^{*}(\bar{h})(\kappa)=j_{1}\left(j_{0}(\bar{h})\right)\left(j_{1}(\kappa)\right)=j_{1}\left(j_{0}(\bar{h})(\kappa)\right)
$$

and $A=j_{0}(\bar{h})(\kappa)$ has cardinality at most $\kappa^{++}$in $M$, using the properties of $\bar{h}$. In particular, since $\kappa^{++}<\lambda$, we have $\lambda \in j_{1}(A)=j_{1}[A]$, which is a contradiction, since $\lambda$ was the critical point of $j_{1}$.

We can conclude that any embedding $j^{*}$ arising from a normal measure on $\kappa$ in $V[g][G]$ is a lift of the ground model $(\kappa+2)$-strongness embedding $j$. But there are exactly $\kappa^{++}$many such lifts: the lift to $V[g]$ is unique, and there are $\kappa^{++}$many possibilities for the final lift to $V[g][G]$, according to item (6). Therefore there are only $\kappa^{++}$many normal measures on $\kappa$ in $V[g][G]$.

Ben-Neria and Gitik [3] have announced that the consistency strength required to achieve the failure of GCH at a measurable cardinal carrying a unique normal measure is exactly that of a measurable cardinal $\kappa$ with $o(\kappa)=\kappa^{++}$. Their method is flexible enough to allow us to incorporate it into our proof of theorem 22 , reducing the consistency strength hypothesis required there from a $(\kappa+2)$-strong cardinal $\kappa$ to just $o(\kappa)=\kappa^{++}$. We have chosen to present the proof based on the original Friedman-Magidor argument since it avoids some complications arising from using the optimal hypotheses

3.3. (Joint) Laver diamonds and the number of normal measures. The only method of controlling the existence of (joint) Laver diamonds we have seen is by controlling the number of large cardinal measures, relying on the rough bound given by Proposition 13. One has to wonder whether merely the existence of sufficiently many measures guarantees the existence of (joint) Laver diamonds. We focus on the simplest form of the question, concerning measurable cardinals.

Question 23. Suppose $\kappa$ is measurable and there are at least $2^{\lambda}$ many normal measures on $\kappa$ for some $\lambda \geq \kappa$. Does there exist a measurable joint Laver sequence for $\kappa$ of length $\lambda$ ?

As the special case when $\lambda=\kappa$, the question includes the possibility that having $2^{\kappa}$ many normal measures, the minimum required, suffices to give the existence of a measurable Laver function for $\kappa$. Even in this very special case it seems implausible that simply having enough measures would automatically yield a Laver function. Nevertheless, in all of the examples of models obtained by forcing and in which we have control over the number of measures that we have seen, Laver functions have existed. On the other hand, Laver functions and joint Laver sequences also exist in canonical inner models that have sufficiently many measures. These models carry long Mitchell-increasing sequences of normal measures that we can use to obtain ordinal-guessing Laver functions. We can then turn these into actual Laver functions by exploiting the structure of these models. 
Definition 24. Let $A$ be a set (or class) of ordinals and let $\bar{\ell}$ be an $A$-guessing Laver function for some large cardinal $\kappa$. Let $\triangleleft$ be some wellorder (one arising from an $L$-like inner model, for example). We say that $\triangleleft$ is suitable for $\bar{\ell}$ if, for any $\alpha \in A$, there is an elementary embedding $j$, witnessing the largeness of $\kappa$, such that $\bar{\ell}$ guesses $\alpha$ via $j$ and $j(\triangleleft) \uparrow(\alpha+1)=\triangleleft \uparrow(\alpha+1)$; that is, the wellorders $j(\triangleleft)$ and $\triangleleft$ agree on their first $\alpha+1$ many elements.

If $\mathcal{J}$ is a class of elementary embeddings witnessing the largeness of $\kappa$, we say that $\triangleleft$ is supersuitable for $\mathcal{J}$ if $j(\triangleleft) \uparrow j(\kappa)=\triangleleft \uparrow j(\kappa)$ for any $j \in \mathcal{J}$.

We could, for example, take the class $\mathcal{J}$ to consist of all ultrapower embeddings by normal measures on $\kappa$ or, more to the point, all ultrapower embeddings arising from a fixed family of extenders. We should also note that, for the notion to make sense, the order type of $\triangleleft$ must be quite high: at least $\sup A$ in the case of wellorders suitable for an $A$-guessing Laver function and at least $\sup _{j \in \mathcal{J}} j(\kappa)$ for supersuitable wellorders (the latter would also make sense if the order type of $\triangleleft$ were smaller than $\kappa$, but that case is not of much interest).

If $\bar{\ell}$ is an ordinal-guessing Laver function and $\mathcal{J}$ is a class of elementary embeddings that includes all the embeddings that $\bar{\ell}$ requires to guess its targets, then any wellorder that is supersuitable for $\mathcal{J}$ is also suitable for $\bar{\ell}$. The following lemma describes the way in which suitable wellorders will be used to turn ordinal-guessing Laver functions into set-guessing ones.

Lemma 25. Let $A$ be a set (or class) of ordinals and let $\bar{\ell}$ be an A-guessing Laver function for some large cardinal $\kappa$. Let $\triangleleft$ be a wellorder such that $\operatorname{otp}(\triangleleft) \subseteq A$. If $\triangleleft$ is suitable for $\bar{\ell}$, then there is a B-guessing Laver function for $\kappa$, where $B$ is the field of $\triangleleft$.

Proof. We can define a $B$-guessing Laver function by simply letting $\ell(\xi)$ be the $\bar{\ell}(\xi)$ th element of $\triangleleft$. Then, given a target $b \in B$, we can find its index $\alpha$ in the wellorder $\triangleleft$ and an embedding $j$ such that $j(\bar{\ell})(\kappa)=\alpha$. Since $\triangleleft$ is suitable for $\bar{\ell}$, the orders $\triangleleft$ and $j(\triangleleft)$ agree on their $\alpha$ th element and so $\ell$ guesses $b$ via $j$.

It follows from the above lemma that in any model with a sufficiently supersuitable wellorder, being able to guess ordinals suffices to be able to guess arbitrary sets.

Lemma 26. Let $X$ be a set (or class) of ordinals and let $\mathcal{J}$ be a class of elementary embeddings of $L[X]$ with critical point $\kappa$ such that $j(X) \cap j(\kappa)=X \cap j(\kappa)$ for any $j \in \mathcal{J}$. Then $\leq_{X}$, the canonical order of $L[X]$, is supersuitable for $\mathcal{J}$.

Proof. This is obvious; the order $\leq_{X}\left\lceil j(\kappa)\right.$ is definable in $L_{j(\kappa)}[X]$, but by our coherence hypothesis this structure is just the same as $L_{j(\kappa)}[j(X)]$.

We are mostly interested in this lemma in the case when $X=\vec{E}$ is an extender sequence and $L[\vec{E}]$ is an extender model in the sense of [22]. In particular, we want $\vec{E}$ to be acceptable (a technical condition which implies enough condensation properties in $L[\vec{E}]$ to conclude $H_{\lambda}^{L[\vec{E}]}=L_{\lambda}[\vec{E}]$ ), coherent (meaning that if $j: L[\vec{E}] \rightarrow$ $L[\vec{F}]$ is an ultrapower by the $\alpha$ th extender of $\vec{E}$ then $\vec{F} \uparrow(\alpha+1)=\vec{E}\lceil\alpha)$, and to use Jensen indexing (meaning that the index of an extender $E$ on $\vec{E}$ with critical point $\kappa$ is $j_{E}(\kappa)^{+}$, as computed in the ultrapower). 
Corollary 27. Let $V=L[\vec{E}]$ be an extender model. Then the canonical wellorder is supersuitable for the class of ultrapower embeddings by the extenders on the sequence $\vec{E}$.

Proof. This is immediate from the preceding lemma and the fact that our extender sequences are coherent and use Jensen indexing.

Theorem 28. Let $V=L[\vec{E}]$ be an extender model. Let $\kappa$ be a cardinal such that every normal measure on $\kappa$ appears on the sequence $\vec{E}$. If $o(\kappa) \geq \kappa^{+}$then $\Delta_{\kappa}^{\text {meas }}$ holds. Moreover, if $o(\kappa)=\kappa^{++}$then $\Delta_{\kappa, \kappa^{+}}^{\text {meas }}$, and even $\triangle_{\kappa}^{\text {meas }}\left(H_{\kappa^{++}}\right)$, holds.

In particular, the above theorem implies $\Delta_{\kappa}^{\text {meas }}$ holds in the least inner model with the required number of measures and the same holds for $\Delta_{\kappa, \kappa^{+}}^{\text {meas }}$. This provides further evidence that the answer to Question 23, which remains open, might turn out to be positive.

Proof. We can argue for the two cases more or less uniformly: let $\lambda \in\left\{\kappa^{+}, \kappa^{++}\right\}$ such that $\lambda \leq o(\kappa)$. The function $\bar{\ell}(\xi)=o(\xi)$ is a $\lambda$-guessing measurable Laver function for $\kappa$. By the acceptability of $\vec{E}$ we have that $H_{\lambda}=L_{\lambda}[\vec{E}]$. The canonical wellorder $\leq_{\vec{E}} \cap L_{\lambda}[\vec{E}]$ has order type $\lambda$ and, by Corollary 27, is supersuitable for the class of ultrapower embeddings by normal measures on $\kappa$. It follows that $\leq_{\vec{E}}$ is suitable for $\bar{\ell}$, so, by Lemma 25 , there is an $H_{\lambda}$-guessing measurable Laver function for $\kappa$.

To finish the proof we still need to produce a joint measurable Laver sequence for $\kappa$, in the case that $o(\kappa)=\kappa^{++}$. This is done in exactly the same way as in Proposition 4; one simply uses the $H_{\kappa^{++}}$-guessing Laver function to guess the whole sequence of targets for a joint Laver sequence.

Interestingly, if we restrict to a smaller set of targets, having enough normal measures does give us Laver functions.

Lemma 29. Let $\kappa$ be a regular cardinal and $\gamma \leq \kappa$ and suppose that $\left\langle\mu_{\alpha} ; \alpha<\gamma\right\rangle$ is a sequence of distinct normal measures on $\kappa$. Then there is a sequence $\left\langle X_{\alpha} ; \alpha<\gamma\right\rangle$ of pairwise disjoint subsets of $\kappa$ such that $X_{\alpha} \in \mu_{\beta}$ if and only if $\alpha=\beta$.

Proof. We prove the lemma by induction on $\gamma$. In the base step, $\gamma=1$, we simply observe that, since $\mu_{0} \neq \mu_{1}$, we must have a set $X_{0} \subseteq \kappa$ such that $X_{0} \in \mu_{0}$ and $\kappa \backslash X_{0} \in \mu_{1}$.

The successor step proceeds similarly. Suppose that the lemma holds for sequences of length $\gamma$ and fix a sequence of measures $\left\langle\mu_{\alpha} ; \alpha<\gamma+1\right\rangle$. By the induction hypothesis we can find pairwise disjoint sets $\left\langle Y_{\alpha} ; \alpha<\gamma\right\rangle$ such that each $Y_{\alpha}$ picks out a unique measure among those with indices below $\gamma$. Again, since $\mu_{\gamma}$ is distinct from all of the other measures, we can find sets $Z_{\alpha} \in \mu_{\gamma} \backslash \mu_{\alpha}$ for each $\alpha<\gamma$. Then the sets $X_{\alpha}=Y_{\alpha} \backslash Z_{\alpha}$ for $\alpha<\gamma$ and $X_{\gamma}=\bigcap_{\alpha<\gamma} Z_{\alpha}$ are as required.

In the limit step suppose that the lemma holds for all $\delta<\gamma$. We can then fix sequences $\left\langle X_{\alpha}^{\delta} ; \alpha<\delta\right\rangle$ for each $\delta<\gamma$ as above. The argument proceeds slightly differently depending on whether $\gamma=\kappa$ or not. If $\gamma<\kappa$ we can simply let $X_{\alpha}=$ $\bigcap_{\alpha<\delta<\gamma} X_{\alpha}^{\delta} \in \mu_{\alpha}$. If, on the other hand, we have $\gamma=\kappa$, then first let $Y_{\alpha}=$ $\triangle_{\alpha<\delta<\kappa} X_{\alpha}^{\delta} \in \mu_{\alpha}$. Given $\alpha<\beta<\gamma$, the sets $Y_{\alpha}$ and $Y_{\beta}$ are almost contained in $X_{\alpha}^{\beta+1}$ and $X_{\beta}^{\beta+1}$, respectively. Since these two are, in turn, disjoint, $Y_{\alpha}$ and $Y_{\beta}$ 
have bounded intersection. Now consider

$$
X_{\alpha}=Y_{\alpha} \backslash \bigcup_{\beta<\alpha}\left(Y_{\alpha} \cap Y_{\beta}\right)
$$

for $\alpha<\kappa$. Since $Y_{\alpha} \cap Y_{\beta}$ is bounded for all $\beta<\alpha$, we still have $X_{\alpha} \in \mu_{\alpha}$. Furthermore, we obviously have $X_{\alpha} \cap X_{\beta}=\emptyset$ for $\beta<\alpha$ and this implies that the $X_{\alpha}$ are pairwise disjoint.

Theorem 30. Let $\kappa$ be a measurable cardinal and $\gamma<\kappa^{+}$an ordinal. There is a $\gamma$-guessing measurable Laver function for $\kappa$ if and only if there are at least $|\gamma|$ many normal measures on $\kappa$.

Proof. First suppose that $\triangle_{\kappa}^{\text {meas }}(\gamma)$ holds. Then, just as in Proposition 13, each target $\alpha<\gamma$ requires its own embedding $j$ via which it is guessed and this gives us $|\gamma|$ many distinct normal measures.

Conversely, suppose that we have at least $|\gamma|$ many normal measures on $\kappa$. We can apply Lemma 29 to find a sequence of pairwise disjoint subsets of $\kappa$ distinguishing these measures. By reorganizing the measures and the distinguishing sets we may assume that they are given in sequences of length $\gamma$. We now have normal measures $\left\langle\mu_{\alpha} ; \alpha<\gamma\right\rangle$ and sets $\left\langle X_{\alpha} ; \alpha<\gamma\right\rangle$ such that $\mu_{\alpha}$ is the unique measure concentrating on $X_{\alpha}$; we may even assume that the $X_{\alpha}$ partition $\kappa$.

Let $f_{\alpha}$ for $\alpha<\gamma$ be the representing functions for $\alpha$, that is, $j\left(f_{\alpha}\right)(\kappa)=\alpha$ for any ultrapower embedding $j$ by a normal measure on $\kappa$. Constructing these functions is not difficult. If $\alpha<\kappa$, we can simply take $f_{\alpha}$ to be the constant function with value $\alpha$. If $\kappa \leq \alpha<\gamma<\kappa^{+}$, we can fix a wellorder $\triangleleft_{\alpha}$ of $\kappa$ in ordertype $\alpha$, and the function $f_{\alpha}(\xi)=\operatorname{otp}\left(\triangleleft_{\alpha} \cap(\xi \times \xi)\right)$ will be a representing function for $\alpha .^{5}$

We can now define a $\gamma$-guessing Laver function $\ell$ by letting $\ell(\xi)=f_{\alpha}(\xi)$ where $\alpha$ is the unique index such that $\xi \in X_{\alpha}$. This function indeed guesses any target $\alpha<\gamma$ : simply let $j: V \rightarrow M$ be the ultrapower by $\mu_{\alpha}$. Since $\mu_{\alpha}$ concentrates on $X_{\alpha}$ we have $j(\ell)(\kappa)=j\left(f_{\alpha}\right)(\kappa)=\alpha$.

Corollary 31. Let $\kappa$ be a measurable cardinal and fix a subset $A \subseteq H_{\kappa^{+}}$of size at most $\kappa$. Then there is an A-guessing measurable Laver function for $\kappa$ if and only if there are at least $|A|$ many normal measures on $\kappa$.

Proof. The forward direction follows just as before: each target in $A$ gives its own normal measure on $\kappa$. Conversely, if there are at least $|A|$ many normal measures on $\kappa$ then, by Theorem 30, there is an $|A|$-guessing measurable Laver function $\bar{\ell}$. Fix a bijection $f:|A| \rightarrow A$. We may assume, moreover, that $A \subseteq \mathcal{P}(\kappa)$. Then we can define an $A$-guessing Laver function $\ell$ by letting $\ell(\xi)=f(\bar{\ell}(\xi)) \cap \xi$. This works: to guess $f(\alpha)$ we let $\bar{\ell}$ guess $\alpha$ via some $j$. Then $j(\ell)(\kappa)=j(f(\alpha)) \cap \kappa=f(\alpha)$.

Lemma 29 can be recast in somewhat different language, giving it, and the subsequent results, a more topological flavour.

Given a cardinal $\kappa$, let $\mathcal{M}(\kappa)$ be the set of normal measures on $\kappa$. We can topologize $\mathcal{M}(\kappa)$ by having, for each $X \subseteq \kappa$, a basic neighbourhood $[X]=\{\mu \in$ $\mathcal{M}(\kappa) ; X \in \mu\}$ (this is just the topology induced on $\mathcal{M}(\kappa)$ by the Stone topology on the space of ultrafilters on $\kappa$ ). Lemma 29 can now be restated to say that any subspace of $\mathcal{M}(\kappa)$ of size at most $\kappa$ is discrete and, moreover, the basic open sets

\footnotetext{
${ }^{5}$ These functions $f_{\alpha}$ are essentially just the first $\kappa^{+}$many canonical functions for $\kappa$, see [13, Section 1.3].
} 
witnessing this can be taken to arise from a pairwise disjoint family of subsets of $\kappa$; such subspaces of spaces of ultrafilters are sometimes also called strongly discrete (see [7], for example). One might thus hope to show the existence of Laver functions by exhibiting even larger discrete subspaces of $\mathcal{M}(\kappa)$. In pursuit of that goal we obtain the following generalization of Corollary 31 .

Theorem 32. Let $\kappa$ be a measurable cardinal and $A \subseteq \mathcal{P}(\kappa)$. Then $\triangle_{\kappa}^{\text {meas }}(A)$ holds if and only if there are for each $a \in A$ a set $S_{a} \subseteq \kappa$ and a normal measure $\mu_{a}$ on $\kappa$ such that $\left\{\mu_{a} ; a \in A\right\}$ is discrete in $\mathcal{M}(\kappa)$, as witnessed by $\left\{S_{a} ; a \in A\right\}$, and $S_{a} \cap S_{b} \subseteq\{\xi ; a \cap \xi=b \cap \xi\}$.

We could have relaxed our hypothesis to $A \subseteq H_{\kappa^{+}}$by working with Mostowski codes.

Proof. Assume first that $\ell$ is a measurable $A$-guessing Laver function for $\kappa$. Then we can let $S_{a}=\{\xi ; \ell(\xi)=a \cap \xi\}$. Obviously we have $j(\ell)(\kappa)=a$ if and only if the measure derived from $j$ concentrates on $S_{a}$. It follows that the measures $\mu_{a}$ derived this way form a discrete subspace of $\mathcal{M}(\kappa)$ and we obviously have $S_{a} \cap S_{b} \subseteq$ $\{\xi ; a \cap \xi=b \cap \xi\}$.

Conversely, assume we have such a discrete family of measures $\mu_{a}$ and a family of sets $S_{a}$ as described. We can define an $A$-guessing measurable Laver function $\ell$ by letting $\ell(\xi)=a \cap \xi$ where $a$ is such that $\xi \in S_{a}$. This is well defined by the coherence condition imposed upon the $S_{a}$, and it is easy to see that $\ell$ satisfies the guessing property.

This topological viewpoint presents a number of questions which might suggest an approach to Question 23. For example, it might be the case that every discrete subset of $\mathcal{M}(\kappa)$ has its discreteness witnessed by a family of sets $S_{a}$ as in Theorem 32. If this were so, we could reduce the problem of finding Laver functions to the seemingly simpler problem of finding large ${ }^{6}$ discrete subspaces of $\mathcal{M}(\kappa)$. But even this simpler task is problematic, since it might be possible that $\mathcal{M}(\kappa)$ has size (at least) $\kappa^{+}$but has no discrete subspaces of size $\kappa^{+}$at all.

3.4. $\Delta_{\kappa}$-trees. Thus far we have thought of joint Laver diamonds as simply matrices or sequences of Laver diamonds. To better facilitate the reflection properties required for forcing iterations using prediction, we would now like a different representation. A reasonable attempt seems to be trying to align the joint Laver sequence with the full binary tree of height $\kappa$.

Definition 33. Let $\kappa$ be a large cardinal supporting a notion of Laver diamond. A $\triangle_{\kappa}$-tree is a labelling of the binary tree such that the labels along the branches form a joint Laver sequence. More precisely, a $\triangle_{\kappa}$-tree is a function $D:{ }^{<\kappa} 2 \rightarrow V$ such that for any sequence of targets $\left\langle a_{s} ; s \in{ }^{\kappa} 2\right\rangle$ there is an elementary embedding $j$, witnessing the largeness $\kappa$, such that $j(D)(s)=a_{s}$ for all $s \in{ }^{\kappa} 2$.

Given an $I \subseteq{ }^{\kappa} 2$, an $I-\triangle_{\kappa}$-tree is a function $D$ as above, satisfying the same guessing property but only for sequences of targets indexed by $I$.

Naturally, we will in this section mostly be interested in $\Delta_{\kappa}^{\theta \text {-sc }}$-trees, that is, $\Delta_{\kappa^{-}}$ trees whose branches form a $\Delta_{\kappa, 2^{\kappa}}^{\theta \text {-sc }}$-sequence. If the degree of supercompactness of $\kappa$ is sufficiently large, then $\triangle_{\kappa}^{\theta \text {-sc }}$-trees are nothing new.

\footnotetext{
${ }^{6}$ Recall that Lemma 29 says that every subset of $\mathcal{M}(\kappa)$ of size $\leq \kappa$ is already strongly discrete.
} 
Proposition 34. Suppose $\kappa$ is $\theta$-supercompact and $\theta \geq 2^{\kappa}$. Then a $\triangle_{\kappa}^{\theta \text {-sc }}$-tree exists if and only if a $\theta$-supercompactness Laver function for $\kappa$ does (if and only if $\Delta_{\kappa, 2^{\kappa}}^{\theta-\mathrm{sc}}$ holds).

Proof. The forward implication is trivial, so we focus on the reverse implication. Let $\ell$ be a Laver function for $\kappa$. For any $t \in{ }^{<\kappa} 2$ define $D(t)=\ell(|t|)(t)$ if this makes sense and $D(t)=\emptyset$ otherwise. We claim this defines a $\triangle_{\kappa}^{\theta \text {-sc }}$-tree. Indeed, let $\vec{a}=\left\langle a_{s} ; s \in{ }^{\kappa} 2\right\rangle$ be a sequence of targets. Since $\theta \geq 2^{\kappa}$ we get $\vec{a} \in H_{\theta^{+}}$, so there is a $\theta$-supercompactness embedding $j$ such that $j(\ell)(\kappa)=\vec{a}$. Therefore, given any $s \in{ }^{\kappa} 2$, we have $j(D)(s)=j(\ell)(\kappa)(s)=a_{s}$

In other situations, however, the existence of a $\triangle_{\kappa}^{\theta \text {-sc }}$-tree can have strictly higher consistency strength than merely a $\theta$-supercompact cardinal.

Definition 35. Let $X$ be a set and $\theta$ a cardinal. A cardinal $\kappa$ is $X$-strong with closure $\theta$ if there is an elementary embedding $j: V \rightarrow M$ with critical point $\kappa$ such that ${ }^{\theta} M \subseteq M$ and $X \in M$.

Proposition 36. Suppose $\kappa$ is $\theta$-supercompact and there is $a \Delta_{\kappa}^{\theta \text {-sc }}$-tree. Then $\kappa$ is $X$-strong with closure $\theta$ for any $X \subseteq H_{\theta^{+}}$of size at most $2^{\kappa}$.

Proof. Suppose $D:{ }^{<\kappa} 2 \rightarrow V_{\kappa}$ is a $\Delta_{\kappa}^{\theta \text {-sc }}$-tree and fix an $X \subseteq H_{\theta^{+}}$of size at most $2^{\kappa}$. Let $f:{ }^{\kappa} 2 \rightarrow X$ enumerate $X$. We can then find a $\theta$-supercompactness embedding $j: V \rightarrow M$ with critical point $\kappa$ such that $j(D)(s)=f(s)$ for all $s \in{ }^{\kappa} 2$. In particular, $X=j(D)\left[{ }^{\kappa} 2\right]$ is an element of $M$, as required.

If $2^{\kappa} \leq \theta$ then $X$-strongness with closure $\theta$ for all $X \subseteq H_{\theta^{+}}$of size $2^{\kappa}$ amounts to just $\theta$-supercompactness and Proposition 34 gives the full equivalence of Laver functions and $\Delta_{\kappa}^{\theta \text {-sc }}$-trees. But if $\theta<2^{\kappa}$, then $X$-strongness with closure $\theta$ can have additional consistency strength. For example, we might choose $X$ to be a normal measure on $\kappa$ to see that $\kappa$ must have nontrivial Mitchell rank (by iterating this idea we can even deduce that $\left.o(\kappa)=\left(2^{\kappa}\right)^{+}\right)$. In the typical scenario where $2^{\kappa}=2^{\theta}=\theta^{+}$, we can also reach higher and choose $X$ to be a normal measure on $\mathcal{P}_{\kappa}(\theta)$ and see that $\kappa$ must also have nontrivial $\theta$-supercompactness Mitchell rank. We can use this observation to show that there might not be any $\triangle_{\kappa}^{\theta \text {-sc }}$-trees, even in the presence of very long joint Laver sequences.

Theorem 37. Suppose GCH holds and let $\kappa$ be $\theta$-supercompact where either $\theta=\kappa$ or $\operatorname{cf}(\theta)>\kappa$. Then there is a cardinal-preserving forcing extension in which $\kappa$ remains $\theta$-supercompact, has a $\theta$-supercompactness joint Laver sequence of length $2^{\kappa}$, but is also the least measurable cardinal. In particular, $\theta<2^{\kappa}$ and there are no $\Delta_{\kappa}^{\theta-s c}$-trees in the extension.

Proof. We may assume by prior forcing, as in the proof of Theorem 8 , that $\kappa$ has a Laver function. Additionally, by performing either Magidor's iteration of Prikry forcing (see [17]) or applying an argument due to Apter and Shelah (see [2]), depending on whether $\theta=\kappa$ or not, we may assume that, in addition to being $\theta$-supercompact, $\kappa$ is also the least measurable cardinal. ${ }^{7}$

\footnotetext{
${ }^{7}$ Of course, if $\theta>\kappa$, this arrangement requires a strong failure of GCH at $\kappa$. In fact, $2^{\kappa}=\theta^{+}$ in the Apter-Shelah model.
} 
We now apply Corollary 10 and arrive at a model where $\kappa$ carries a $\theta$-supercompactness joint Laver sequence of length $2^{\kappa}$, but is also the least measurable cardinal. It follows that there can be no $\Delta_{\kappa}^{\theta \text {-sc }}$-trees (or even $\Delta_{\kappa}^{\text {meas }}$-trees), since, by the discussion above, their existence would imply that $\kappa$ has nontrivial Mitchell rank, implying that there are many measurables below $\kappa$.

Proposition 36 can be improved slightly to give a jump in consistency strength even for $I$ - $\triangle_{\kappa}^{\theta \text {-sc }}$-trees where $I$ is not the whole set of branches. A simple modification of the proof given there yields the following result, together with the corresponding version of Theorem 37 .

Theorem 38. Suppose $\kappa$ is $\theta$-supercompact and there is an $I-\Delta_{\kappa}^{\theta \text {-sc }}$-tree for some $I \subseteq{ }^{\kappa} 2$ of size $2^{\kappa}$. If $I$ is definable (with parameters) over $H_{\kappa^{+}}$then $\kappa$ is $X$-strong with closure $\theta$ for any $X \subseteq H_{\theta^{+}}$of size at most $2^{\kappa}$.

The above theorem notwithstanding, we shall give a construction which shows that the existence of an $I-\triangle_{\kappa}^{\theta \text {-sc }}$-tree does not yield additional consistency strength, provided that we allow $I$ to be sufficiently foreign to $H_{\kappa^{+}}$. The argument will rely on being able to surgically alter a Cohen subset of $\kappa^{+}$in a variety of ways. To this end we fix some notation beforehand.

Definition 39. Let $f$ and $g$ be functions. The graft of $f$ onto $g$ is the function $g \imath f$, defined on $\operatorname{dom}(g)$ by

$$
(g \prec f)(x)= \begin{cases}f(x) ; \quad x \in \operatorname{dom}(g) \cap \operatorname{dom}(f) \\ g(x) ; \quad x \in \operatorname{dom}(g) \backslash \operatorname{dom}(f)\end{cases}
$$

Essentially, the graft replaces the values of $g$ with those of $f$ on their common domain.

Lemma 40. Let $\lambda$ be a regular cardinal and assume $\nabla_{\lambda}$ holds. Suppose $M$ is a transitive model of ZFC (either set- or class-sized) such that $\lambda \in M$ and $M^{<\lambda} \subseteq M$ and $\left|\mathcal{P}(\lambda)^{M}\right|=\lambda$. Then there are an unbounded set $I \subseteq \lambda$ and a function $g: \lambda \rightarrow$ $H_{\lambda}$ such that, given any $f: I \rightarrow H_{\lambda}$, the graft $g$ ? $f$ is generic for $\operatorname{Add}(\lambda, 1)$ over $M{ }^{8}$

The hypothesis of $\nabla_{\lambda}$ is often automatically satisfied. Specifically, our assumptions about $M$ imply that $2^{<\lambda}=\lambda$. If $\lambda=\kappa^{+}$is a successor, this gives $2^{\kappa}=\kappa^{+}$which already implies $\nabla_{\lambda}$ by a result of Shelah [20].

We are grateful to Joel David Hamkins for suggesting this proof.

Proof. Let $\left\langle f_{\alpha} ; \alpha<\lambda\right\rangle$, with $f_{\alpha}: \alpha \rightarrow H_{|\alpha|}$, be a $\diamond_{\lambda}$-sequence and fix an enumeration $\left\langle D_{\alpha} ; \alpha<\lambda\right\rangle$ of the open dense subsets of $\operatorname{Add}(\lambda, 1)$ in $M$. We shall construct by recursion a descending sequence of conditions $p_{\alpha} \in \operatorname{Add}(\lambda, 1)$ and an increasing sequence of sets $I_{\alpha}$ as approximations to $g$ and $I$. Specifically, we shall use $\diamond_{\lambda}$ to guess pieces of any potential function $f$ and ensure along the way that the modified conditions $p_{\alpha} 2 f$ meet all of the listed dense sets.

Suppose we have built the sequences $\left\langle p_{\alpha} ; \alpha<\gamma\right\rangle$ and $\left\langle I_{\alpha} ; \alpha<\gamma\right\rangle$ for some $\gamma<\lambda$. Let $I_{\gamma}^{*}=\bigcup_{\alpha<\gamma} I_{\alpha}$. Let $p_{\gamma}^{*} \in M$ be an extension of $\bigcup_{\alpha<\gamma} p_{\alpha}$ such that $I_{\gamma}^{*} \subseteq \operatorname{dom}\left(p_{\gamma}^{*}\right) \in \lambda$; such an extension exists in $M$ by our assumption on the closure of $M$.

${ }^{8}$ Here we take the version of $\operatorname{Add}(\lambda, 1)$ which adds a function $g: \lambda \rightarrow H_{\lambda}$ by initial segments. 
Let us briefly summarize the construction. We shall surgically modify the condition $p_{\gamma}^{*}$ by grafting the function given by $\diamond_{\lambda}$ onto it. We shall then extend this modified condition to meet one of our dense sets, after which we will undo the surgery. We will be left with a condition $p_{\gamma}$ which is one step closer to ensuring that the result of one particular grafting $g$ ? $f$ is generic. At the same time we also extend $I_{\gamma}^{*}$ by adding a point beyond the domains of all the conditions constructed so far.

More precisely, let $\tilde{p}_{\gamma}^{*}=p_{\gamma}^{*} 2\left(f_{\gamma}\left\lceil I_{\gamma}^{*}\right)\right.$. This is still a condition in $M$. Let $\widetilde{p}_{\gamma}$ be any extension of this condition inside $D_{\eta_{\gamma}}$, where $\eta_{\gamma}$ is the least such that $\tilde{p}_{\gamma}^{*} \notin D_{\eta_{\gamma}}$, and satisfying $\operatorname{dom}\left(\widetilde{p}_{\gamma}\right) \in \lambda$. Finally, we undo the initial graft and set $p_{\gamma}=\widetilde{p}_{\gamma} 2\left(p_{\gamma}^{*}\left\lceil I_{\gamma}^{*}\right)\right.$. Note that we have $p_{\gamma} \leq p_{\gamma}^{*}$. We also extend our approximation to $I$ with the first available point, letting $I_{\gamma}=I_{\gamma}^{*} \cup\left\{\min \left(\lambda \backslash \operatorname{dom}\left(p_{\gamma}\right)\right)\right\}$.

Once we have completed this recursive construction we can set $I=\bigcup_{\gamma<\lambda} I_{\gamma}$ and $g=\bigcup_{\gamma<\lambda} p_{\gamma}$. Let us check that these do in fact have the desired properties.

Let $f: I \rightarrow H_{\lambda}$ be a function. We need to show that $g$ < $f$ is generic over $M$. Using $\diamond_{\lambda}$, we find that there are stationarily many $\gamma$ such that $f_{\gamma}=f\lceil\gamma$. Note also that there are club many $\gamma$ such that $I_{\gamma}^{*} \subseteq \gamma$ is unbounded, and together this means that $S=\left\{\gamma ; f_{\gamma}\left\lceil I_{\gamma}^{*}=f\lceil(I \cap \gamma)\}\right.\right.$ is stationary. The conditions $\widetilde{p}_{\gamma}$ for $\gamma \in S$ extend each other and we have $\bigcup_{\gamma \in S} \widetilde{p}_{\gamma}=g \imath f$. Furthermore, since the sets $D_{\alpha}$ are open, the construction of $\widetilde{p}_{\gamma}$ ensures that eventually these conditions will meet all of these dense sets, showing that $g \imath f$ really is generic.

The construction in the above proof is quite flexible and can be modified to make the set $I$ generic in various ways as well (for example, we can arrange for $I$ to be Cohen or dominating over $M$, and have other similar properties).

Theorem 41. If $\kappa$ is $\theta$-supercompact, then there is a forcing extension in which there is an $I-\Delta_{\kappa}^{\theta \text {-sc }}$-tree for some $I \subseteq{ }^{\kappa} 2$ of size $2^{\kappa}$.

Proof. If $\theta \geq 2^{\kappa}$ then even a single Laver function for $\kappa$ gives rise to a full $\triangle_{\kappa}$-tree, by Proposition 34, and we can force the existence of a Laver function by Theorem 8 . We thus focus on the remaining case when $\kappa \leq \theta<2^{\kappa}$.

We make similar simplifying assumptions as in Theorem 8. Just as there we assume that $\theta=\theta^{<\kappa}$. Furthermore, we may assume that $2^{\theta}=\theta^{+}$, since this can be forced without affecting the $\theta$-supercompactness of $\kappa$. Note that these cardinal arithmetic hypotheses imply that $2^{\kappa}=\theta^{+}$.

Let $\mathbb{P}$ be the length $\kappa$ Easton support iteration which adds, in a recursive fashion, a labelling of the tree ${ }^{<\kappa} 2$ of the extension. Specifically, let $\mathbb{P}$ force with $\mathbb{Q}_{\gamma}=$ $\operatorname{Add}\left(2^{\gamma}, 1\right)$ at each inaccessible $\gamma<\kappa$ stage $\gamma$. Let $G \subseteq \mathbb{P}$ be generic and let $G_{\gamma}$ be the piece added at stage $\gamma$. Using suitable coding, we can see each $G_{\gamma}$, in $V[G]$, as a function $G_{\gamma}:{ }^{\gamma} 2 \rightarrow H_{\gamma^{+}}$; in particular, $G_{\gamma}$ really does label the whole level ${ }^{\gamma} 2$ in the final extension, since no new nodes appear in the tree ${ }^{\leq \gamma} 2$ after stage $\gamma$ in the iteration $\mathbb{P}$. Thus $G$ induces a map $D:{ }^{<\kappa} 2 \rightarrow V_{\kappa}[G]$, by extending the $G_{\gamma}$ in any way we like to the entire tree. We shall show that $D$ is an $I-\Delta_{\kappa}^{\theta \text {-sc }}$-tree for some specifically chosen $I$.

Fix a $\theta$-supercompactness embedding $j: V \rightarrow M$ in $V$. Note that $M[G]^{\theta} \subseteq$ $M[G]$ in $V[G]$ as well, since the forcing $\mathbb{P}$ is $\theta^{+}$-cc. Furthermore, in $V[G]$ we still have $2^{\theta}=\theta^{+}$, which implies $\nabla_{\theta^{+}}$by a result of Shelah [20]. We also know that $\left|\mathcal{P}\left(\theta^{+}\right)^{M[G]}\right|=\theta^{+}$, since $|j(\kappa)|=\theta^{+}$and $\left|\mathcal{P}\left(\theta^{+}\right)^{M[G]}\right|<j(\kappa)$ because $\theta<j(\kappa)$ and 
$j(\kappa)$ is inaccessible in $M[G]$. Now apply Lemma 40 to $M[G]$ and $\lambda=\theta^{+}$to obtain an $I \subseteq{ }^{\kappa} 2$ of size $\theta^{+}$and a function $g:{ }^{\kappa} 2 \rightarrow H_{\theta^{+}}$such that for any $f: I \rightarrow H_{\theta^{+}}$in $V[G]$, the graft $g$ ? $f$ is generic over $M[G]$. We claim that $D$ is an $I-\triangle_{\kappa}^{\theta \text {-sc }}$-tree.

To check the guessing property, fix a sequence of targets $\vec{a}=\left\langle a_{s} ; s \in I\right\rangle$ in $V[G]$. We shall lift the embedding $j$ to $V[G]$. Let us write $j(\mathbb{P})=\mathbb{P} * \mathbb{Q}_{\kappa} * \mathbb{P}_{\text {tail }}$. We know that $g \imath \vec{a}$ is $M[G]$-generic for $\mathbb{Q}_{\kappa}$, so we only need to find the further generic for $\mathbb{P}_{\text {tail }}$. We easily see that $M[G][g<\vec{a}]^{\theta} \subseteq M[G][g<\vec{a}]$ in $V[G]$, that $\mathbb{P}_{\text {tail }}$ is $\leq \theta$-closed in that model, and that $M[G][g<\vec{a}]$ only has $\theta^{+}$-many subsets of $\mathbb{P}_{\text {tail }}$. We can thus diagonalize against these dense sets in $\theta^{+}$-many steps and produce a generic $G_{\text {tail }}$ for $\mathbb{P}_{\text {tail }}$. Putting all of this together, we can lift $j$ to $j: V[G] \rightarrow M[j(G)]$ in $V[G]$, where $j(G)=G *(g \prec \vec{a}) * G_{\text {tail }}$. Now consider $j(D)$. This is exactly the labelling of the tree ${ }^{<j(\kappa)} 2$ in $M[j(G)]$ given by $j(G)$. Furthermore, for any $s \in I$, we have $j(D)(s)=(g \prec \vec{a})(s)=a_{s}$, verifying the guessing property.

Given a $\Delta_{\kappa}$-tree, it is easy to produce a joint Laver sequence of length $2^{\kappa}$ from it by just reading the labels along each branch of the tree. The resulting sequence then exhibits a large degree of coherence. We might wonder about the possibility of reversing this process, starting with a joint Laver sequence and attempting to fit it into a tree. But, taken literally, this property is not very robust. For example, all functions in such a joint Laver sequence must have the same value at 0 , so this coherence property of joint Laver sequences can be destroyed without changing the sequence in any essential way. To avoid such trivialities, we relax the definition to only ask for coherence modulo bounded perturbations.

Definition 42. Let $\kappa$ be a regular cardinal and $\vec{f}=\left\langle f_{\alpha} ; \alpha<\lambda\right\rangle$ a sequence of functions defined on $\kappa$. The sequence $\vec{f}$ is treeable if there are a bijection $e: \lambda \rightarrow{ }^{\kappa} 2$ and a tree labelling $D:{ }^{<\kappa} 2 \rightarrow V$ such that, for all $\alpha<\lambda$, we have $D(e(\alpha) \uparrow \xi)=$ $f_{\alpha}(\xi)$ for all but boundedly many $\xi<\kappa$.

Lemma 43. Let $\kappa$ be a regular cardinal and assume that $2^{<\kappa}<2^{\kappa}$. Let $G \subseteq$ $\operatorname{Add}\left(\kappa, 2^{\kappa}\right)$ be generic. Then $G$ is not treeable.

Proof. Let us write $G=\left\langle g_{\alpha} ; \alpha<2^{\kappa}\right\rangle$ as a sequence of its slices. Now suppose that this sequence were treeable and let $\dot{e}$ and $\dot{D}$ be names for the indexing function and the labelling of ${ }^{<\kappa} 2$, respectively. Our cardinal arithmetic assumption implies that the name $\dot{D}$ only involves conditions from a bounded part of the poset $\operatorname{Add}\left(\kappa, 2^{\kappa}\right)$, so we may assume that the labelling $D$ exists already in the ground model. Let $p$ be an arbitrary condition and $\alpha<\kappa$. Since we assumed that $G$ was forced to be treeable, there is a name $\dot{\gamma}$ for an ordinal beyond which $G_{\alpha}$ agrees with $D\lceil f(\alpha)$. By strengthening $p$ if necessary, we may assume that the value of $\dot{\gamma}$ has been decided. We now inductively construct a countable descending sequence of conditions below $p$, deciding longer and longer initial segments of $\dot{e}(\alpha)$, in such a way that, for some $\delta>\gamma$, their union $p^{*} \leq p$ decides $\dot{e}(\alpha)\left\lceil\delta\right.$ but does not decide $G_{\alpha}(\delta)$. Then $p^{*}$ can be further extended to a condition forcing $G_{\alpha}(\delta) \neq D(\dot{e}(\alpha)\lceil\delta)$, which contradicts the fact that $p$ forces that $G$ is treeable.

Corollary 44. If $\kappa$ is $\theta$-supercompact, then there is a forcing extension in which there is a nontreeable $\theta$-supercompactness joint Laver sequence for $\kappa$ of length $2^{\kappa}$.

Proof. The joint Laver sequence constructed in Theorem 8 was added by forcing with $\operatorname{Add}\left(\kappa, 2^{\kappa}\right)$, so it is not treeable by Lemma 43. 


\section{JOINT LAVER DIAMONDS FOR STRONG CARDINALS}

Definition 45. A function $\ell: \kappa \rightarrow V_{\kappa}$ is a $\theta$-strongness Laver function if it guesses elements of $V_{\theta}$ via $\theta$-strongness embeddings with critical point $\kappa$.

If $\kappa$ is fully strong then a function $\ell: \kappa \rightarrow V_{\kappa}$ is a Laver function for $\kappa$ if it is a $\theta$-strongness Laver function for $\kappa$ for all $\theta$.

Just as in the supercompact case, we shall say that $\Delta_{\kappa}^{\theta \text {-str }}$ holds if there is a $\theta$ strongness Laver function for $\kappa$; since our default set of targets in this case is $V_{\theta}$, this is just the same as $\Delta_{\kappa}^{\theta \text {-str }}\left(V_{\theta}\right)$. In the case of full strongness, we shall similarly say that $\triangle_{\kappa}^{\text {str }}$ holds if there is a strongness Laver function for $\kappa$, which should be read as $\triangle_{\kappa}^{\text {str }}(V)$.

A similar factoring argument as in the supercompact case shows that we can afford to be imprecise about which embeddings we count as $\theta$-strongness embeddings in the definition above. Specifically, if $j: V \rightarrow M$ is any $\theta$-strongness embedding with critical point $\kappa$ and a function $\ell$ guesses a target $a \in V_{\theta}$ via $j$, then $\ell$ also guesses $a$ via the induced $\left(\kappa, V_{\theta}\right)$-extender ultrapower embedding.

As in the supercompact case, $2^{\kappa}$ is the largest possible cardinal length of a $\theta$ strongness joint Laver sequence for $\kappa$, just because there are only $2^{\kappa}$ many functions $\ell: \kappa \rightarrow V_{\kappa}$.

The set of targets $V_{\theta}$ is a bit unwieldy and lacks some basic closure properties, particularly in the case when $\theta$ is a successor ordinal. The following lemma shows that, modulo some coding, we can recover a good deal of closure under sequences.

Lemma 46. Let $\theta$ be an infinite ordinal and let $I \in V_{\theta}$ be a set. If $\theta$ is successor ordinal or $\operatorname{cf}(\theta)>|I|$ then $V_{\theta}$ is closed under a coding scheme for sequences indexed by I. Moreover, this coding is $\Delta_{0}$-definable.

Proof. If $\theta=\omega$ then the $I$ under consideration are finite. Since $V_{\omega}$ is already closed under finite sequences we need only deal with $\theta>\omega$.

Fix in advance a simply definable flat pairing function $[\cdot, \cdot]$ (flat in the sense that any infinite $V_{\alpha}$ is closed under it; the Quine-Rosser pairing function will do).

Let $\vec{a}=\left\langle a_{i} ; i \in I\right\rangle$ be a sequence of elements of $V_{\theta}$. For each $i \in I$ we can find an (infinite) ordinal $\theta_{i}<\theta$ such that $a_{i} \cup\{i\} \subseteq V_{\theta_{i}}$. Now let $\widetilde{a}_{i}=\left\{[i, b] ; b \in a_{i}\right\} \subseteq V_{\theta_{i}}$ and finally define $\widetilde{a}=\bigcup_{i \in I} \widetilde{a}_{i}$. We see that $\widetilde{a} \subseteq V_{\sup _{i} \theta_{i}}$ and, under our hypotheses, $\sup _{i} \theta_{i}<\theta$. It follows that $\widetilde{a} \in V_{\left(\sup _{i} \theta_{i}\right)+1} \subseteq V_{\theta}$ as required.

Proposition 47. Let $\kappa$ be $\theta$-strong with $\kappa+2 \leq \theta$ and let $\lambda \leq 2^{\kappa}$ be a cardinal. If there is a $\theta$-strongness Laver function for $\kappa$ and $\theta$ is either a successor ordinal or $\lambda<\operatorname{cf}(\theta)$ then there is a $\theta$-strongness joint Laver sequence of length $\lambda$ for $\kappa$.

In particular, if $\theta$ is a successor, then a single $\theta$-strongness Laver function already yields a joint Laver sequence of length $2^{\kappa}$, the maximal possible.

Proof. We aim to imitate the proof of Proposition 4. To that end, fix an $I \subseteq \mathcal{P}(\kappa)$ of size $\lambda$ and a bijection $f: \lambda \rightarrow I$. If $\ell$ is a Laver function for $\kappa$, we define a joint Laver sequence by letting $\ell_{\alpha}(\xi)$, for each $\alpha<\lambda$, be the element of $\ell(\xi)$ with index $f(\alpha) \cap \xi$ in the coding scheme described in Lemma 46.

It is now easy to verify that the functions $\ell_{\alpha}$ form a joint Laver sequence: given a sequence of targets $\vec{a}$, we can replace it with a coded version $\widetilde{a} \in V_{\theta}$, by using $f$ and Lemma 46 . We can then use $\ell$ to guess $\widetilde{a}$ and the $\theta$-strongness embedding obtained this way will witness the joint guessing property of the $\ell_{\alpha}$. 
Again, as in the supercompact case, if the Laver diamond we started with works for several different $\theta$ then the joint Laver sequence derived above will also work for those same $\theta$. In particular, if $\kappa$ is strong, then combining the argument from Proposition 47 with the construction of a strongness Laver function due to Gitik and Shelah [9] gives an analogue of Corollary 5.

Corollary 48. If $\kappa$ is strong then there is a strongness joint Laver sequence for $\kappa$ of length $2^{\kappa}$.

Proposition 47 implies that in most cases (that is, for most $\theta$ ) we do not need to do any additional work beyond ensuring that there is a $\theta$-strongness Laver function for $\kappa$ to automatically also find the longest possible joint Laver sequence. For example, if $\theta$ is a successor or if $\operatorname{cf}(\theta)>2^{\kappa}$ then a single $\theta$-strongness Laver function yields a joint Laver sequence of length $2^{\kappa}$. To gauge the consistency strength of the existence of $\theta$-strongness joint Laver sequences for $\kappa$ we should therefore only focus on the consistency strength required for a single Laver diamond, and, separately, on $\theta$ of low cofinality.

Forcing constructions for a single $\theta$-strongness Laver diamond are known. However, since we weren't able to find a suitable reference, we give the proofs in some detail.

We are going to need an analogue of Lemma 7 for strong cardinals.

Definition 49. A $\theta$-strongness Menas function for a cardinal $\kappa$ is a function $f: \kappa \rightarrow$ $\kappa$ such that there is a $\theta$-strongness embedding $j: V \rightarrow M$ with $\operatorname{cp}(j)=\kappa$ and $j(f)(\kappa)=\theta$.

We should mention that our definition differs slightly from Hamkins' original definition in [10], where he says that $f$ is a $\theta$-strongness Menas function if $j(f)(\kappa)>$ $\beth_{\theta}$ for some $\theta$-strongness embedding $j$. A Menas function in our sense gives rise to one in Hamkins' original sense, and is in general more convenient to work with.

Lemma 50. If $\kappa$ is $\theta$-strong, then $\kappa$ carries a $\theta$-strongness Menas function.

Proof. We define a function $f: \kappa \rightarrow \kappa$ by letting $f(\alpha)=0$ if $\alpha$ is $\kappa$-strong, and otherwise let $f(\alpha)$ be the least $\gamma<\kappa$ such that $\alpha$ is not $\gamma$-strong. An argument much like the one in Lemma 7 shows that we can find a $\theta$-strongness embedding $j: V \rightarrow M$ with critical point $\kappa$ such that $\kappa$ is not $\theta$-strong in $M$, and that $j(f)(\kappa)=\theta$ for this $j$.

Lemma 51. Let $\kappa$ be a cardinal and suppose $\mathbb{P} \subseteq V_{\kappa}$ is a poset. Let $G \subseteq \mathbb{P}$ be generic and assume that $\kappa$ is a $\beth$-fixed point in $V[G]$. For any ordinal $\alpha \geq \kappa+1$, every element of $V[G]_{\alpha}=\left(V_{\alpha}\right)^{V[G]}$ has a $\mathbb{P}$-name coded in $V_{\alpha}$.

Proof. We argue by induction on $\alpha$. In the base step, the key point is that, since $\kappa$ is a $\beth$-fixed point in $V[G]$, names for elements of $V[G]_{\kappa+1}$ can be replaced with names for subsets of $\kappa$. But nice names for subsets of $\kappa$ are essentially just subsets of $\kappa \times \mathbb{P}$ and are thus elements of $V_{\kappa+1}$. The limit step of the induction is trivial, so it only remains for us to consider the successor step.

Assume that every element of $V[G]_{\alpha}$, for some $\alpha \geq \kappa+1$, has a name coded in $V_{\alpha}$. Now consider an arbitrary element of $V[G]_{\alpha+1}$. By the induction hypothesis, it has a nice name of the form $\sigma=\bigcup_{x}\{x\} \times A_{x}$, where each $A_{x}$ is an antichain in $\mathbb{P}$ and the union runs over all the coded names $x$ in $V_{\alpha}$. We can think of $\sigma$ as simply the sequence of the antichains $A_{x}$, indexed by a subset of $V_{\alpha}$, and, because each 
$A_{x}$ is an element of $V_{\kappa+1} \subseteq V_{\alpha}$, Lemma 46 implies that this sequence is coded by an element of $V_{\alpha+1}$.

Lemma 52. Let $\kappa$ be a cardinal and let $\mathbb{P}=\operatorname{Add}\left(\kappa^{+}, 1\right)$. Let $G \subseteq \mathbb{P}$ be generic. For any ordinal $\alpha \geq \kappa$, every element of $V[G]_{\alpha}=\left(V_{\alpha}\right)^{V[G]}$ has a $\mathbb{P}$-name coded in $V_{\alpha}$.

Proof. We can argue much like in the proof we just gave, by induction on $\alpha$. For $\alpha=\kappa$ or $\alpha=\kappa+1$, no new elements of $V_{\alpha}$ are added by $\mathbb{P}$, so we can simply take check names. The limit step of the induction is trivial, so it only remains for us to consider the successor step.

Assume that every element of $V[G]_{\alpha}$, for some $\alpha \geq \kappa+2$, has a name coded in $V_{\alpha}$. We may as well work with an isomorphic copy of the poset $\mathbb{P}$ which is a subset of $V_{\kappa+1}$. This means that every antichain of $\mathbb{P}$ is an element of $V_{\kappa+2} \subseteq V_{\alpha}$. Now consider an arbitrary element of $V[G]_{\alpha+1}$. By the induction hypothesis, it has a nice name of the form $\sigma=\bigcup_{x}\{x\} \times A_{x}$, where each $A_{x}$ is an antichain in $\mathbb{P}$, and the union runs over all the coded names in $V_{\alpha}$ for elements of $(V[G])_{\alpha}$. As in the previous proof, we think of $\sigma$ as a sequence of antichains indexed by a subset of $V_{\alpha}$, and use Lemma 46 to obtain a code for $\sigma$ in $V_{\alpha+1}$.

Theorem 53. If $\kappa$ is $\theta$-strong and $\theta$ is either a successor ordinal or $\operatorname{cf}(\theta) \geq \kappa^{+}$, then there is a $\left(2^{\kappa}\right)^{+}$-cc forcing extension in which there is a $\theta$-strongness Laver function for $\kappa$. In the extension $2^{\kappa}=\kappa^{+}$holds, $\kappa^{+}$is preserved, and, if $\theta$ was a limit ordinal, $\operatorname{cf}(\theta) \geq \kappa^{+}$remains true.

Proof. If $\theta \leq \kappa$, no forcing is necessary, and the case $\theta=\kappa+1$ was essentially covered by Theorem 8 . We may therefore restrict our attention to the case when $\theta \geq \kappa+2$.

Fix a Menas function $f$ as in Lemma 50 and define the forcing $\mathbb{P}_{\kappa}$ as the length $\kappa$ Easton support iteration which forces with $\mathbb{Q}_{\gamma}=\operatorname{Add}\left(\gamma^{+}, 1\right)$ at inaccessible closure points $\gamma$ of the function $f$. Let $\mathbb{P}=\mathbb{P}_{\kappa} * \mathbb{Q}_{\kappa}$ and let $G=G_{\kappa} * g \subseteq \mathbb{P}$ be generic over $V$. Let us first show that $\kappa$ remains $\theta$-strong in $V[G]$. We will describe later how to derive a Laver function in the extension.

By the definition of the Menas function $f$, we can fix a $\theta$-strongness extender embedding $j: V \rightarrow M$ such that $j(f)(\kappa)=\theta$. We can write $j(\mathbb{P})=\mathbb{P} * \mathbb{P}_{\text {tail }} * j\left(\mathbb{Q}_{\kappa}\right)$.

Because of our assumptions about $\theta$, Lemma 46 implies that $M$ is in fact closed under $\kappa$-sequences. Since $\mathbb{P}_{\kappa}$ is $\kappa$-cc, the model $M\left[G_{\kappa}\right]$ remains closed under $\kappa$ sequences in $V\left[G_{\kappa}\right]$, and, since $\mathbb{Q}_{\kappa}$ is $\leq \kappa$-closed, this remains true for $M[G]$ in $V[G]$.

Recall that every element of $M[G]$ is of the form $j(F)(a)^{G}$ for some $a \in V_{\theta}$ and some function $F \in V$ defined on $V_{\kappa}$. Since $\mathbb{P}_{\text {tail }}$ has size $j(\kappa)$ in $M[G]$, every open dense subset of $\mathbb{P}_{\text {tail }}$ can be represented in this way by using a function $F: V_{\kappa} \rightarrow$ $V_{\kappa+1}$. For a fixed $F$ like this, let $\mathcal{D}_{F}$ be the collection of all open dense subsets of $\mathbb{P}_{\text {tail }}$ of the form $j(F)(a)^{G}$ for some $a \in V_{\theta}$. Then $\mathcal{D}_{F} \in M[G]$ and it has size $\beth_{\theta}$ in $M[G]$. Since the first stage of forcing in $\mathbb{P}_{\text {tail }}$ occurs after the first inaccessible

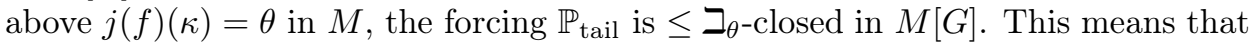
$D_{F}=\bigcap \mathcal{D}_{F}$ is a dense subset of $\mathbb{P}_{\text {tail }}$. Since $2^{\kappa}=\kappa^{+}$in $V[G]$ (because of the last stage of forcing), there are only $\kappa^{+}$many of these dense sets $D_{F}$, counted in $V[G]$. Therefore we can line them up and, using the closure of the poset $\mathbb{P}_{\text {tail }}$ and of the model $M[G]$, meet all of them in order to build a generic $G_{\text {tail }} \in V[G]$ over $M[G]$. This allows us to lift the embedding $j$ to $j: V\left[G_{\kappa}\right] \rightarrow M[G]\left[G_{\text {tail }}\right]$. 
The final lift is easier: since $\mathbb{Q}_{\kappa}$ is $\leq \kappa$-distributive, the filter $h$ generated by the image $j[g]$ is generic over $M[G]\left[G_{\text {tail }}\right]$ and allows us to lift to $j: V[G] \rightarrow$ $M[G]\left[G_{\text {tail }}\right][h]$. To see that this embedding witnesses the $\theta$-strongness of $\kappa$ in $V[G]$, we use Lemmas 51 and 52 . Lemma 51 implies that every element of $V\left[G_{\kappa}\right]_{\theta}$ has a name coded in $V_{\theta}$, so, since $V_{\theta} \in M$ and $G_{\kappa} \in M[G]$, we get $V\left[G_{\kappa}\right]_{\theta} \in M[G] \subseteq$ $M[G]\left[G_{\text {tail }}\right][h]$. Lemma 52 , in turn, says that every element of $V[G]_{\theta}$ has a name coded in $V\left[G_{\kappa}\right]_{\theta}$, and so, because $g \in M[G]$, we get $V[G]_{\theta} \in M[G] \subseteq M[G]\left[G_{\text {tail }}\right][h]$. This shows that $\kappa$ is $\theta$-strong in $V[G]$.

Now let us turn our attention to the Laver function. We define a function $\ell \in V[G]$ on $\kappa$ by letting $\ell(\xi)$ be the set whose Mostowski code appears as the first set coded in the sequence of bits $G_{\xi^{\prime}}$, where $\xi^{\prime}$ is the least inaccessible closure point of $f$ above $\xi$. It follows from this definition that, given a $\theta$-strongness embedding $j$ in $V[G]$, we evaluate $j(\ell)(\kappa)$ by consulting the first slice of the generic $G_{\text {tail }}$, as constructed above, and seeing what is coded there. To show that $\ell$ really is a Laver function, it only remains for us to see that any target $a \in V[G]_{\theta}$ can be coded into $G_{\text {tail }}$ appropriately. But this is straightforward: if we start with a $\theta$ strongness embedding in $V$ as above and proceed with the lifting argument, we saw that $V[G]_{\theta} \subseteq M[G]$, so the target $a$ appears in $M[G]$. Consequently, the bit sequence of the Mostowski code of $a$ is a condition in the first stage of forcing in $\mathbb{P}_{\text {tail }}$. If we now run our construction of $G_{\text {tail }}$ as described above, with the added requirement that we start with the fixed condition coding $a$ before we meet all of the dense sets $D_{F}$, we will obtain a generic $G_{\text {tail }}$ whose first slice codes exactly $a$, and a lifted embedding $j$ satisfying $j(\ell)(\kappa)=a$. Therefore $\ell$ really is a $\theta$-strongness Laver function in $V[G]$.

Combining Theorem 53 with Proposition 47, we immediately obtain the following corollary.

Corollary 54. Let $\kappa$ be $\theta$-strong with $\kappa+2 \leq \theta$. If $\theta$ is either a successor ordinal or $\operatorname{cf}(\theta)>2^{\kappa}$ then there is a forcing extension in which $\Delta_{\kappa, 2^{\kappa}}^{\theta-\operatorname{str}}$ holds.

Again, the forcing we do in Theorem 53 will collapse $2^{\kappa}$ to $\kappa^{+}$, so the conclusion $\Delta_{\kappa, 2^{\kappa}}^{\theta-\text { itr }}$ in this corollary should be read with the parameters evaluated in the extension.

Moving on to the case of $\theta$ of low cofinality, it is important to note that Theorem 53 has little to say in this situation. In fact we do not know whether one can force the existence of a $\theta$-strongness Laver function when $\theta$ is a limit ordinal of low cofinality, starting from just a $\theta$-strong cardinal. Of course, one can do it starting from just a little bit more, like a $(\theta+1)$-strong cardinal, but it is unclear what the sharpest result is. But, since we are interested in jointness phenomena, let us gloss over this issue and ask whether the hypotheses in Proposition 47 are really necessary in the singular case.

Question 55. Suppose there is a $\theta$-strongness Laver function for $\kappa$ (with $\theta$ possibly being a limit of low cofinality). Is there a $\theta$-strongness joint Laver sequence of length $\kappa$ ? Or even of length $\omega$ ?

We give a partial answer to this question. In contrast to the supercompact case, some restrictions are in fact necessary to allow for the existence of joint Laver sequences for $\theta$-strong cardinals. The existence of even the shortest of such sequences can surpass the existence of a $\theta$-strong cardinal in consistency strength. 
To give a better lower bound on the consistency strength required, we introduce a notion of Mitchell rank for $\theta$-strong cardinals, inspired by Carmody [4].

Definition 56. Let $\kappa$ be a cardinal and $\theta$ an ordinal. The $\theta$-strongness Mitchell order $\triangleleft$ for $\kappa$ is defined on the set of $\left(\kappa, V_{\theta}\right)$-extenders, by letting $E \triangleleft F$ if $E$ is an element of the (transitive collapse of the) ultrapower of $V$ by $F$.

Unsurprisingly, this Mitchell order has properties analogous to those of the usual Mitchell order on normal measures on $\kappa$ or the $\theta$-supercompactness Mitchell order on normal fine measures on $\mathcal{P}_{\kappa}(\theta)$, as studied by Carmody. In particular, the $\theta$ strongness Mitchell order is well-founded and gives rise to a notion of a $\theta$-strongness Mitchell rank. Having $\theta$-strongness Mitchell rank at least 2 implies that many cardinals below $\kappa$ have reflected versions of $\theta$-strongness; for example, if $\kappa$ has $(\kappa+$ $\omega)$-strongness Mitchell rank at least 2, then there are stationarily many cardinals $\lambda<\kappa$ which are $(\lambda+\omega)$-strong (and much more is true).

We should mention a bound on the $\theta$-strongness Mitchel rank of a cardinal $\kappa$. If $j: V \rightarrow M$ is the ultrapower by a $\left(\kappa, V_{\theta}\right)$-extender then any $\left(\kappa, V_{\theta}\right)$-extenders in $M$ appear in $V_{j(\kappa)}^{M}$. It follows that these extenders can be written in the from $j(f)(a)$ for some function $f: V_{\kappa} \rightarrow V_{\kappa}$ and a seed $a \in V_{\theta}$. In particular, there are at most $\beth_{\theta}$ many such extenders, counted in $V$. Any given extender therefore has at most $\beth_{\theta}$ many predecessors in the Mitchell order, so the highest possible $\theta$-strongness Mitchell rank of $\kappa$ is $\beth_{\theta}^{+}$.

Theorem 57. Let $\kappa$ be a $\theta$-strong cardinal, where $\theta$ is a limit ordinal, and $\operatorname{cf}(\theta) \leq$ $\kappa<\theta$. If there is a $\theta$-strongness joint Laver sequence for $\kappa$ of length $\operatorname{cf}(\theta)$ then $\kappa$ has maximal $\theta$-strongness Mitchell rank.

Proof. We first show that the existence of a short $\theta$-strongness joint Laver sequence implies a certain degree of hypermeasurability for $\kappa$. Let $\vec{\ell}=\left\langle\ell_{\alpha} ; \alpha<\operatorname{cf}(\theta)\right\rangle$ be the joint Laver sequence. If $\vec{a}=\left\langle a_{\alpha} ; \alpha<\operatorname{cf}(\theta)\right\rangle$ is any sequence of targets in $V_{\theta}$ there is, by definition, a $\theta$-strongness embedding $j: V \rightarrow M$ with critical point $\kappa$ such that $j\left(\ell_{\alpha}\right)(\kappa)=a_{\alpha}$. But we can recover $\vec{\ell}$ from $j(\vec{\ell})$ as an initial segment, since $\vec{\ell}$ is so short. Therefore we actually get the whole sequence $\vec{a} \in M$, just by evaluating that initial segment at $\kappa$. Now consider any $a \subseteq V_{\theta}$. We can resolve $a$ into a $\operatorname{cf}(\theta)$-sequence of elements $a_{\alpha}$ of $V_{\theta}$, by taking a cofinal sequence $\left\langle\theta_{\alpha} ; \alpha<\operatorname{cf}(\theta)\right\rangle$ in $\theta$ and letting $a_{\alpha}=a \cap V_{\theta_{\alpha}}$. It follows that $a=\bigcup_{\alpha} a_{\alpha}$. We can take the $a_{\alpha}$ as our targets for $\vec{\ell}$. Our argument then implies that there is a $\theta$-strongness embedding $j: V \rightarrow M$ so that $\left\langle a_{\alpha} ; \alpha<\operatorname{cf}(\theta)\right\rangle \in M$, and therefore also $a \in M$.

Now let $E$ be an arbitrary $\left(\kappa, V_{\theta}\right)$-extender. Since $E$ can be represented as a family of measures on $\kappa$ indexed by $V_{\theta}$, it is coded by a subset of $V_{\theta}$ (using the coding scheme from Lemma 46, for example). Applying the argument above, there is a $\theta$-strongness embedding $j: V \rightarrow M$ with critical point $\kappa$ such that $E \in M$. It follows that $\kappa$ is $\theta$-strong in $M$, giving $\kappa$ nontrivial $\theta$-strongness Mitchell rank in $V$.

The argument in fact yields more: given any collection of at most $\beth_{\theta}$ many $\left(\kappa, V_{\theta}\right)$-extenders, we can code the whole family by a subset of $V_{\theta}$ and, again, obtain an extender whose ultrapower contains the entire collection we started with. It follows that, given any family of at most $\beth_{\theta}$ many extenders, we can find an extender which is above all of them in the $\theta$-strongness Mitchell order. Applying this fact inductively now shows that $\kappa$ must have maximal $\theta$-strongness Mitchell rank. 
Again, we remind the reader that we have not determined the consistency strength of the existence of a $\theta$-strongness Laver function for $\theta$ of low cofinality. It may well be that the high Mitchell rank we just derived from $\Delta_{\kappa, \mathrm{cf}(\kappa)}^{\theta-\operatorname{str}}$ can already be obtained from just $\triangle_{\kappa}^{\theta \text {-str }}$.

Just as in the case of $\theta$-supercompactness we can also consider $\Delta_{\kappa}^{\theta \text {-str }}$-trees. In view of Propositions 47 and 34 it is not surprising that again, for most $\theta$, a single $\theta$-strongness Laver diamond yields a $\Delta_{\kappa}^{\theta \text {-str }}$-tree.

Proposition 58. Suppose $\kappa$ is $\theta$-strong where $\kappa+2 \leq \theta$ and $\theta$ is either a successor ordinal or $\operatorname{cf}(\theta)>2^{\kappa}$. Then $a \Delta_{\kappa}^{\theta \text {-str }}$-tree exists if and only if a $\theta$-strongness Laver function for $\kappa$ does (if and only if $\Delta_{\kappa, 2^{\kappa}}^{\theta-\mathrm{sc}}$ holds).

Proof. We follow the proof of Proposition 34. Note that, since $\theta \geq \kappa+2$, we have ${ }^{\kappa} 2 \in V_{\theta}$, so $V_{\theta}$ is closed under sequences indexed by ${ }^{\kappa} 2$ via the coding scheme given by Lemma 46 . If $\ell$ is a $\theta$-strongness Laver function for $\kappa$ we define a $\Delta_{\kappa}^{\theta \text {-str }}$-tree by letting $D(t)$ be the element with index $t$ in the sequence coded by $\ell(|t|)$, if this makes sense. It is now easy to check that this truly is a $\Delta_{\kappa}^{\theta \text {-str }}$-tree: given any sequence of targets $\vec{a}$ we simply use the Laver function $\ell$ to guess it (or, rather, its code), and the embedding $j$ obtained this way will witness the guessing property for $D$.

\section{JOINT DIAMONDS}

Motivated by the joint Laver sequences of the previous sections, we now apply the jointness concept to smaller cardinals. Of course, since we do not have any elementary embeddings of the universe with critical point $\omega_{1}$, say, we need a reformulation that will make sense in this setting as well.

Consider a measurable Laver function $\ell$ and let $a \subseteq \kappa$. By definition there is an elementary embedding $j: V \rightarrow M$ such that $j(\ell)(\kappa)=a$. Let $U$ be the normal measure on $\kappa$ derived from this embedding. Since $U$ is normal, $a$ is represented in the ultrapower by the function $f_{a}(\xi)=a \cap \xi$, and thus, by Łoś's theorem, we conclude that $\ell(\xi)=a \cap \xi$ for $U$-almost all $\xi$. In particular, the set of such $\xi$ is stationary in $\kappa$. Therefore $\ell$ is (essentially) a $\nabla_{\kappa}$-sequence. Similarly, if we are dealing with a joint Laver sequence $\left\langle\ell_{\alpha} ; \alpha<\lambda\right\rangle$ there is for every sequence $\left\langle a_{\alpha} ; \alpha<\lambda\right\rangle$ of subsets of $\kappa$ a normal measure on $\kappa$ with respect to which the set $\left\{\xi<\kappa ; \ell_{\alpha}(\xi)=a_{\alpha} \cap \xi\right\}$ has measure one for each $\alpha$.

This understanding of jointness seems amenable to transfer to smaller cardinals. There are still no normal measures on $\omega_{1}$, but perhaps we can weaken that requirement slightly.

Recall that a filter on a regular cardinal $\kappa$ is normal if it is closed under diagonal intersections, and uniform if it contains all the cobounded sets. It is a standard result that the club filter on $\kappa$ is the least normal uniform filter on $\kappa$ (in fact, a normal filter is uniform iff it extends the club filter). It follows that any subset of $\kappa$ contained in a (proper) normal uniform filter is stationary. Conversely, if $S \subseteq \kappa$ is stationary, then it is easy to check that $S$, together with the club filter, generates a normal uniform filter on $\kappa$. Altogether, we see that a set is stationary iff it is contained in a normal uniform filter. This observation suggests an analogy between Laver functions and $\nabla_{\kappa}$-sequences: in the same way that Laver functions 
guess their targets on large sets with respect to some large cardinal measure, $\nabla_{\kappa^{-}}$ sequences guess their targets on large sets with respect to some normal uniform filter. Extending the analogy, in the same way that a joint Laver sequence is a collection of Laver functions that guess sequences of targets on large sets with respect to a common large cardinal measure (corresponding to the single embedding $j$ ), a collection of $\nabla_{\kappa}$-sequences will be joint if they guess sequences of targets on large sets with respect to a common normal uniform filter.

We will adopt the following terminology: if $\kappa$ is a cardinal then a $\kappa$-list is a function $d: \kappa \rightarrow \mathcal{P}(\kappa)$ with $d(\alpha) \subseteq \alpha$ (this term seems to have originated in Weiß' dissertation [21]).

Definition 59. Let $\kappa$ be an uncountable regular cardinal. A $\otimes_{\kappa, \lambda}$-sequence is a sequence $\vec{d}=\left\langle d_{\alpha} ; \alpha<\lambda\right\rangle$ of $\kappa$-lists such that for every sequence $\left\langle a_{\alpha} ; \alpha<\lambda\right\rangle$ of subsets of $\kappa$ there is a (proper) normal uniform filter $\mathcal{F}$ on $\kappa$ such that for every $\alpha$ the guessing set $S_{\alpha}=S\left(d_{\alpha}, a_{\alpha}\right)=\left\{\xi<\kappa ; d_{\alpha}(\xi)=a_{\alpha} \cap \xi\right\}$ is in $\mathcal{F}$.

An alternative, apparently simpler attempt at defining jointness would be to require that all the $\kappa$-lists in the sequence guess their respective targets on the same stationary set. Let us say that a $\widehat{ }_{\kappa, \lambda}$-sequence is consonant if for any sequence of targets $\left\langle a_{\alpha} ; \alpha<\lambda\right\rangle$ there is a stationary set $S$ so that $S \subseteq S_{\alpha}$ for all $\alpha<\lambda$.

It is not hard to see that we can derive a consonant $\widehat{\vartheta}_{\kappa, \lambda}$-sequence from a $\nabla_{\kappa^{-}}$ sequence, provided that $\lambda<\kappa$. We omit the proof, since the following proposition shows that the consonance requirement is, in the end, too strong to yield a useful notion of jointness for longer sequences.

Proposition 60. Let $\kappa$ be an uncountable regular cardinal. There are no consonant $\widehat{\vartheta}_{\kappa, \kappa}$-sequences.

Proof. Suppose that $\vec{d}$ is a $\widehat{\vartheta}_{\kappa, \kappa}$-sequence and consider the sequence of targets $\left\langle\kappa \backslash d_{\alpha}(\alpha) ; \alpha<\kappa\right\rangle$. If $\vec{d}$ were consonant, there would definitely need to be some $\xi<\kappa$ so that each particular $\kappa$-list $d_{\alpha}$ guessed its target at $\xi$. But we picked the targets in such a way that $d_{\xi}(\xi)$ is not a good guess for the $\xi$ th target. Therefore $\vec{d}$ is not consonant.

Upon reflection we thus abandon the consonance requirement and insist only on the jointness property as originally stated in the definition. To be sure, every $\Delta_{\kappa, \lambda}$-sequence is also (essentially) a $\widehat{\vartheta}_{\kappa, \lambda}$-sequence. This means that, at least in the presence of sufficient large cardinals, $\otimes_{\kappa, \lambda}$-sequences exist. But, as we will see later in Theorem 66, $\vartheta_{\kappa, \lambda}$-sequences can exist quite independently of large cardinals.

We will not use the following proposition going forward, but it serves to give another parallel between $\diamond$-sequences and Laver diamonds. It turns out that $\diamond$ sequences can be seen as Laver functions, except that they work with generic elementary embeddings.

Proposition 61. Let $\kappa$ be an uncountable regular cardinal and $d$ a $\kappa$-list. Then $d$ is a $\diamond_{\kappa}$-sequence iff there is, for any $a \subset \kappa$, a generic elementary embedding $j: V \rightarrow M$ with critical point $\kappa$ and $M$ wellfounded up to $\kappa+1$ such that $j(d)(\kappa)=a$.

Proof. Suppose $d$ is a $\nabla_{\kappa}$-sequence and fix a target $a \subseteq \kappa$. Let $S(d, a)=\{\xi<$ $\kappa ; d(\xi)=a \cap \xi\}$ be the guessing set. By our discussion above, there is a normal uniform filter $\mathcal{F}$ on $\kappa$ with $S \in \mathcal{F}$. Let $G$ be a generic ultrafilter extending $\mathcal{F}$ and 
$j: V \rightarrow M$ the generic ultrapower by $G$. Then $M$ is wellfounded up to $\kappa^{+}$and $\kappa=[\mathrm{id}]_{G}$. Since $S \in G$, Łoś's theorem now implies that $j(d)(\kappa)=a$.

Conversely, fix a target $a \subseteq \kappa$ and suppose that there is a generic embedding $j$ with the above properties. We can replace $j$ with the induced normal ultrapower embedding and let $U$ be the derived ultrafilter in the extension. Since $j(d)(\kappa)=a$ it follows that $S(d, a) \in U$. But since $U$ extends the club filter, $S(d, a)$ must be stationary.

The same proof will also show that a sequence of $\kappa$-lists is joint if they can guess any sequence of targets via a single generic elementary embedding.

The following key lemma gives a "bottom up" criterion deciding when a collection of subsets of $\kappa$ (namely, some guessing sets) is contained in a normal uniform filter. It is completely analogous to the finite intersection property characterizing containment in a filter.

Definition 62. Let $\kappa$ be an uncountable regular cardinal. A family $\mathcal{A} \subseteq \mathcal{P}(\kappa)$ has the diagonal intersection property if for any function $f: \kappa \rightarrow \mathcal{A}$ the diagonal intersection $\triangle_{\alpha<\kappa} f(\alpha)$ is stationary.

Lemma 63. Let $\kappa$ be uncountable and regular and let $\mathcal{A} \subseteq \mathcal{P}(\kappa)$. The family $\mathcal{A}$ is contained in a normal uniform filter on $\kappa$ iff $\mathcal{A}$ satisfies the diagonal intersection property.

Proof. The forward direction is clear, so let us focus on the converse. Consider the family of sets

$$
E=\left\{C \cap \underset{\alpha<\kappa}{\triangle} f(\alpha) ; C \subseteq \kappa \text { is club and } f \in{ }^{\kappa} \mathcal{A}\right\} .
$$

We claim that $E$ is directed under diagonal intersections: any diagonal intersection of $\kappa$ many elements of $E$ contains another element of $E$. To see this, take $C_{\alpha} \cap$ $\triangle_{\beta<\kappa} f_{\alpha}(\beta) \in E$ for $\alpha<\kappa$. Let $\langle\cdot, \cdot\rangle$ be a pairing function and define $F: \kappa \rightarrow \lambda$ by $F(\langle\alpha, \beta\rangle)=f_{\alpha}(\beta)$. A calculation then shows that

$$
\left.\underset{\alpha<\kappa}{\triangle}\left(C_{\alpha} \cap \triangle_{\beta<\kappa} f_{\alpha}(\beta)\right)=\triangle_{\alpha} C_{\alpha} \cap \triangle_{\alpha} \triangle_{\beta} f_{\alpha}(\beta) \supseteq \underset{\alpha}{\triangle} C_{\alpha} \cap D\right) \cap \triangle_{\alpha} F(\alpha),
$$

where $D$ is the club of closure points of the pairing function.

It follows that closing $E$ under supersets yields a normal uniform filter on $\kappa$. By considering constant functions $f$ we also see that every $a \in \mathcal{A}$ is in this filter.

Lemma 63 will be the crucial tool for verifying $\bigotimes_{\kappa, \lambda}$. More specifically, we shall often apply the following corollary.

Corollary 64. A sequence $\vec{d}=\left\langle d_{\alpha} ; \alpha<\lambda\right\rangle$ is a $\otimes_{\kappa, \lambda}$-sequence iff every subsequence of length $\kappa$ is a $\otimes_{\kappa, \kappa}$-sequence.

Proof. The forward implication is obvious; let us check the converse. Let $\vec{a}=$ $\left\langle a_{\alpha} ; \alpha<\lambda\right\rangle$ be a sequence of targets and let $S_{\alpha}$ be the corresponding guessing sets. By Lemma 63 we need to check that the family $\mathcal{S}=\left\{S_{\alpha} ; \alpha<\lambda\right\}$ satisfies the diagonal intersection property. So fix a function $f: \kappa \rightarrow \lambda$ and consider the diagonal intersection $\triangle_{\alpha} S_{f(\alpha)}$. We may assume without loss of generality that $f$ is injective. By our assumption $\vec{d} \uparrow f[\kappa]$ is a $\diamond_{\kappa, \kappa}$-sequence, so $\left\{S_{f(\alpha)} ; \alpha<\kappa\right\}$ is contained in a normal uniform filter, which means that $\triangle_{\alpha} S_{f(\alpha)}$ is stationary. 
This characterization leads to fundamental differences between joint diamonds and joint Laver diamonds. While the definition of joint diamonds was inspired by large cardinal phenomena, the absence of a suitable analogue of the diagonal intersection property in the large cardinal setting provides for some very surprising results.

Definition 65. Let $\kappa$ be an uncountable regular cardinal. A $\nabla_{\kappa}$-tree is a function $D:{ }^{<\kappa} 2 \rightarrow \mathcal{P}(\kappa)$ such that for any sequence $\left\langle a_{s} ; s \in{ }^{\kappa} 2\right\rangle$ of subsets of $\kappa$ there is a (proper) normal uniform filter on $\kappa$ containing all the guessing sets $S_{s}=S\left(D, a_{s}\right)=$ $\left\{\xi<\kappa ; D\left(s\lceil\xi)=a_{s} \cap \xi\right\}\right.$.

This definition clearly imitates the definition of $\triangle_{\kappa}$-trees. We also have a correspondence in the style of Proposition 61: a $\nabla_{\kappa}$-tree acts like a $\Delta_{\kappa}$-tree using generic elementary embeddings.

The following theorem, the main result of this section, shows that, in complete contrast to our experience with joint Laver diamonds, $\nabla_{\kappa}$ already implies all of its joint versions.

Theorem 66. Let $\kappa$ be an uncountable regular cardinal. The following are equivalent:

(1) $\nabla_{\kappa}$

(2) $\otimes_{\kappa, \kappa}$

(3) $\otimes_{\kappa, 2^{\kappa}}$

(4) There exists a $\diamond_{\kappa}$-tree.

Proof. For the implication (1) $\Longrightarrow(2)$, let $d: \kappa \rightarrow \mathcal{P}(\kappa)$ be a $\diamond_{\kappa}$-sequence and fix a bijection $f: \kappa \rightarrow \kappa \times \kappa$. Define

$$
d_{\alpha}(\xi)=\{\eta<\alpha ;(\alpha, \eta) \in f[d(\xi)]\} .
$$

We claim that $\left\langle d_{\alpha} ; \alpha<\kappa\right\rangle$ is a $\otimes_{\kappa, \kappa}$-sequence.

To see this, take a sequence of targets $\left\langle a_{\alpha} ; \alpha<\lambda\right\rangle$ and let $S_{\alpha}=\left\{\xi<\kappa ; d_{\alpha}(\xi)=\right.$ $\left.a_{\alpha} \cap \xi\right\}$ be the guessing sets. The set

$$
T=\left\{\xi<\kappa ; d(\xi)=f^{-1}\left[\bigcup_{\alpha<\kappa}\{\alpha\} \times a_{\alpha}\right] \cap \xi\right\}
$$

is stationary in $\kappa$. Let $\mathcal{F}$ be the filter generated by the club filter on $\kappa$ together with $T$. This is clearly a proper filter. To see that it is also normal, consider some typical elements $C_{\alpha} \cap T$ of $\mathcal{F}$, where $C_{\alpha} \subseteq \kappa$ is club for each $\alpha<\kappa$. Then $\triangle_{\alpha<\kappa}\left(C_{\alpha} \cap T\right)=\left(\triangle_{\alpha<\kappa} C_{\alpha}\right) \cap T$ is also clearly an element of $\mathcal{F}$.

We claim that we have $S_{\alpha} \in \mathcal{F}$ for all $\alpha<\kappa$, so that $\mathcal{F}$ witnesses the defining property of a $\widehat{\vartheta}_{\kappa, \kappa}$-sequence. Since $f[\xi]=\xi \times \xi$ for club many $\xi<\kappa$, the set

$$
T^{\prime}=\left\{\xi<\kappa ; d(\xi)=f^{-1}\left[\bigcup_{\alpha<\kappa}\{\alpha\} \times a_{\alpha}\right] \cap f^{-1}[\xi \times \xi]\right\}
$$

is just the intersection of $T$ with a club, and is therefore in $\mathcal{F}$. But now observe that

$$
\begin{aligned}
T^{\prime} & =\left\{\xi<\kappa ; d(\xi)=f^{-1}\left[\bigcup_{\alpha<\xi}\{\alpha\} \times\left(a_{\alpha} \cap \xi\right)\right]\right\} \\
& =\left\{\xi<\kappa ; \forall \alpha<\xi: d_{\alpha}(\xi)=a_{\alpha} \cap \xi\right\}=\triangle_{\alpha<\kappa} S_{\alpha} .
\end{aligned}
$$


We see that $T^{\prime} \in \mathcal{F}$ is, modulo a bounded set, contained in each $S_{\alpha}$ and can thus conclude, since $\mathcal{F}$ is uniform, that $S_{\alpha} \in F$ for all $\alpha<\kappa$.

Instead of proving $(2) \Longrightarrow(3)$, it will be easier to show $(2) \Longrightarrow(4)$ directly. Since the implications $(4) \Longrightarrow(3) \Longrightarrow(1)$ are obvious, this will finish the proof.

Fix a $\widehat{ }_{\kappa, \kappa}$-sequence $\vec{d}$. We proceed to construct the $\diamond_{\kappa}$-tree $D$ level by level; in fact the only meaningful work will take place at limit levels. At a limit stage $\gamma<\kappa$ we shall let the first $\gamma$ many $\diamond_{\kappa}$-sequences anticipate the labels and their positions. Concretely, consider the sets $d_{\alpha}(\gamma)$ for $\alpha<\gamma$. For each $\alpha$ we interpret $d_{2 \alpha}(\gamma)$ as a node on the $\gamma$-th level of ${ }^{<\kappa} 2$ and let $D\left(d_{2 \alpha}(\gamma)\right)=d_{2 \alpha+1}(\gamma)$, provided that there is no interference between the different $\nabla_{\kappa}$-sequences. If it should happen that for some $\alpha \neq \beta$ we get $d_{2 \alpha}(\gamma)=d_{2 \beta}(\gamma)$ but $d_{2 \alpha+1}(\gamma) \neq d_{2 \beta+1}(\gamma)$ we scrap the whole level and move on with the construction higher in the tree. At the end we extend $D$ to be defined on the nodes of ${ }^{<\kappa} 2$ that were skipped along the way in any way we like.

We claim that the function $D$ thus constructed is a $\nabla_{\kappa}$-tree. To check this, let us fix a sequence of targets $\vec{a}=\left\langle a_{s} ; s \in{ }^{\kappa} 2\right\rangle$ and let $S_{s}$ be the guessing sets. By Lemma 63 it now suffices to check that $\triangle_{\alpha<\kappa} S_{s_{\alpha}}$ is stationary for any sequence of branches $\left\langle s_{\alpha} ; \alpha<\kappa\right\rangle$.

For $\alpha<\kappa$ let $T_{2 \alpha}=\left\{\xi ; s_{\alpha}^{-1}[\{1\}] \cap \xi=d_{2 \alpha}(\xi)\right\}$ and $T_{2 \alpha+1}=\left\{\xi ; a_{s_{\alpha}} \cap \xi=\right.$ $\left.d_{2 \alpha+1}(\xi)\right\}$. Since our construction was guided by a $\otimes_{\kappa, \kappa}$-sequence, there is a normal uniform filter on $\kappa$ which contains every $T_{\alpha}$. In particular, $T=\triangle_{\alpha<\kappa} T_{\alpha}$ is stationary. By a simple bootstrapping argument, there is a club $C$ of limit ordinals $\gamma$ such that all $s_{\alpha}\lceil\gamma$ for $\alpha<\gamma$ are distinct. Let $\gamma \in C \cap T$. We now have $s_{\alpha}^{-1}[\{1\}] \cap \gamma=d_{2 \alpha}(\gamma)$ and $a_{s_{\alpha}} \cap \gamma=d_{2 \alpha+1}(\gamma)$ for all $\alpha<\gamma$. But this means precisely that the construction of $D$ goes through at level $\gamma$ and that $\gamma \in \bigcap_{\alpha<\gamma} S_{s_{\alpha}}$, and it follows that $C \cap T \subseteq \triangle_{\alpha<\kappa} S_{s_{\alpha}}$, so $\triangle_{\alpha<\kappa} S_{s_{\alpha}}$ is stationary.

We can again consider the treeability of joint diamond sequences, as we did in Definition 42. We get the following analogue of Corollary 44 .

Theorem 67. If $\kappa$ is an uncountable regular cardinal and $G C H$ holds, then after forcing with $\operatorname{Add}\left(\kappa, 2^{\kappa}\right)$ there is a nontreeable $\widehat{\vartheta}_{\kappa, 2^{\kappa}}$-sequence.

Proof. Let $\mathbb{P}=\operatorname{Add}\left(\kappa, 2^{\kappa}\right)$ and $G \subseteq \mathbb{P}$ generic; we refer to the $\alpha$-th subset added by $G$ as $G_{\alpha}$. We will show that the generic $G$, seen as a sequence of $2^{\kappa}$ many

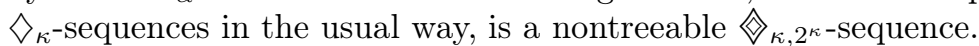

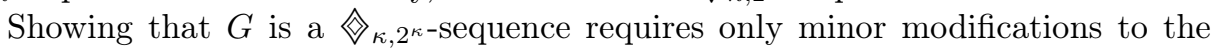
usual proof that a Cohen subset of $\kappa$ codes a $\nabla_{\kappa}$-sequence. Thus, we view each $G_{\alpha}$ as a $\kappa$-list. Fix a sequence $\left\langle\dot{a}_{\alpha} ; \alpha<2^{\kappa}\right\rangle$ of names for subsets of $\kappa$, a name $\dot{f}$ for a function from $\kappa$ to $2^{\kappa}$ and a name $\dot{C}$ for a club in $\kappa$ as well as a condition $p \in \mathbb{P}$. We will find a condition $q \leq p$ forcing that $\dot{C} \cap \triangle_{\alpha<\kappa} S_{\dot{f}(\alpha)}$ is nonempty, where $S_{\dot{f}(\alpha)}$ names the set $\left\{\xi<\kappa ; \dot{a}_{\dot{f}(\alpha)} \cap \xi=G_{\alpha}(\xi)\right\}$; this will show that $G$ codes

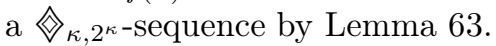

We build the condition $q$ in $\omega$ many steps. To start with, let $p_{0}=p$ and let $\gamma_{0}$ be an ordinal such that $\operatorname{dom}\left(p_{0}\right) \subseteq 2^{\kappa} \times \gamma_{0}$. By deciding more and more of the function $\dot{f}$, the targets $\dot{a}_{\dot{f}(\alpha)}$, and the club $\dot{C}$, we now inductively find ordinals $\gamma_{n}<\delta_{n}<\gamma_{n+1}$, sets $B_{n}^{\alpha} \subseteq \gamma_{n}$, functions $f_{n}$ and a descending sequence of conditions $p_{n}$ satisfying $\operatorname{dom}\left(p_{n}\right) \subseteq 2^{\kappa} \times \gamma_{n}$ and $p_{n+1} \Vdash \delta_{n} \in \dot{C}$ as well as $p_{n+1} \Vdash \dot{f}\left\lceil\delta_{n}=f_{n}\right.$ and $p_{n+1} \Vdash \dot{a}_{f_{n}(\alpha)}=B_{n}^{\alpha}$ for $\alpha<\delta_{n}$. Let $\gamma_{\omega}=\sup _{n} \gamma_{n}=\sup _{n} \delta_{n}$ and $p_{\omega}=$ 
$\bigcup_{n} p_{n}$ and $f_{\omega}=\bigcup_{n} f_{n}$ and $B_{\omega}^{\alpha}=\bigcup_{n} B_{n}^{\alpha}$. The construction of these ensures that $\operatorname{dom}\left(p_{\omega}\right) \subseteq 2^{\kappa} \times \gamma_{\omega}$ and $p_{\omega}$ forces that $\dot{f}\left\lceil\gamma_{\omega}=f_{\omega}\right.$ and $\dot{a}_{\dot{f}(\alpha)} \cap \gamma_{\omega}=B_{\omega}^{\alpha}$ for $\alpha<\gamma_{\omega}$ as well as $\gamma_{\omega} \in \dot{C}$. To obtain the final condition $q$ we now simply extend $p_{\omega}$ by placing the code of $B_{\omega}^{\alpha}$ on top of the $f(\alpha)$-th column for all $\alpha<\gamma_{\omega}$. It now follows immediately that $q \Vdash \gamma_{\omega} \in \dot{C} \cap \triangle_{\alpha<\kappa} S_{\dot{f}(\alpha)}$.

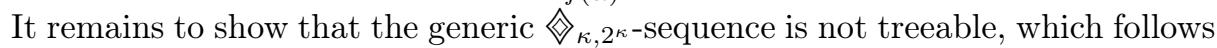
immediately from Lemma 43.

In the case of Laver diamonds we were able to produce models with quite long joint Laver sequences but no $\triangle_{\kappa}$-trees simply on consistency strength grounds (see Theorem 37). In other words, we have models where there are long joint Laver sequences, but none of them are treeable. The situation seems different for ordinary diamonds, as Theorem 66 tells us that treeable joint diamond sequences exist as soon as a single diamond sequence exists. While Theorem 67 shows that it is at least consistent that there are nontreeable such sequences, we should ask whether this is simply always the case.

Question 68. Is it consistent for a fixed $\kappa$, for example $\kappa=\omega_{1}$, that every $\bigotimes_{\kappa, 2^{\kappa}-}$ sequence is treeable? Is it consistent that all $\otimes_{\kappa, 2^{\kappa}}$-sequences are treeable for all $\kappa$ ?

\section{REFERENCES}

1. Arthur W. Apter, James Cummings, and Joel David Hamkins, Large cardinals with few measures, Proceedings of the American Mathematical Society 135 (2007), no. 7, 2291-2300. MR 2299507

2. Arthur W. Apter and Saharon Shelah, Menas' result is best possible, Transactions of the American Mathematical Society 349 (1997), no. 5, 2007-2034. MR 1370634

3. Omer Ben-Neria and Moti Gitik, A model with a unique normal measure on $\kappa$ and $2^{\kappa}=\kappa^{++}$ from optimal assumptions, preprint.

4. Erin Carmody, Force to change large cardinal strength, Ph.D. thesis, The Graduate Center, City University of New York, 2015, ProQuest/UMI Publication No. 3704311.

5. Paul Corazza, Laver sequences for extendible and super-almost-huge cardinals, The Journal of Symbolic Logic 64 (1999), no. 3, 963-983. MR 1779746

6. James Cummings, Iterated forcing and elementary embeddings, Handbook of set theory. Vols. 1, 2, 3, Springer, Dordrecht, 2010, pp. 775-883. MR 2768691

7. Alan Dow, Good and OK ultrafilters, Transactions of the American Mathematical Society 290 (1985), no. 1, 145-160. MR 787959

8. Sy-David Friedman and Menachem Magidor, The number of normal measures, The Journal of Symbolic Logic 74 (2009), no. 3, 1069-1080. MR 2548481

9. Moti Gitik and Saharon Shelah, On certain indestructibility of strong cardinals and a question of Hajnal, Archive for Mathematical Logic 28 (1989), no. 1, 35-42. MR 987765

10. Joel David Hamkins, The lottery preparation, Annals of Pure and Applied Logic 101 (2000), no. 2-3, 103-146. MR 1736060

11. _ A class of strong diamond principles, preprint, arXiv:math/0211419 [math.LO], 2002.

12. Extensions with the approximation and cover properties have no new large cardinals, Fundamenta Mathematicae 180 (2003), no. 3, 257-277. MR 2063629

13. Thomas Jech, Stationary sets, Handbook of set theory. Vols. 1, 2, 3, Springer, Dordrecht, 2010, pp. 93-128. MR 2768680

14. Akihiro Kanamori, Perfect-set forcing for uncountable cardinals, Annals of Mathematical Logic 19 (1980), no. 1-2, 97-114. MR 593029

15. — The higher infinite, second ed., Springer Monographs in Mathematics, SpringerVerlag, Berlin, 2008. MR 2731169

16. Richard Laver, Making the supercompactness of $\kappa$ indestructible under $\kappa$-directed closed forcing, Israel Journal of Mathematics 29 (1978), no. 4, 385-388. MR 0472529 
17. Menachem Magidor, How large is the first strongly compact cardinal? or A study on identity crises, Annals of Mathematical Logic 10 (1976), no. 1, 33-57. MR 0429566

18. - On the existence of nonregular ultrafilters and the cardinality of ultrapowers, Transactions of the American Mathematical Society 249 (1979), no. 1, 97-111. MR 526312

19. Telis K. Menas, On strong compactness and supercompactness, Annals of Mathematical Logic 7 (1974/75), 327-359. MR 0357121

20. Saharon Shelah, Diamonds, Proceedings of the American Mathematical Society 138 (2010), no. 6, 2151-2161. MR 2596054

21. Christoph Weiß, Subtle and ineffable tree properties, Ph.D. thesis, Ludwig-MaximiliansUniversität München, 2010.

22. Martin Zeman, Inner models and large cardinals, De Gruyter Series in Logic and its Applications, vol. 5, Walter de Gruyter \& Co., Berlin, 2002. MR 1876087

Faculty of Information Technology, Czech Technical University in Prague, Thákurova 9, 16000 Praha 6, Czech Republic \& Department of logic, Faculty of Arts, Charles University, nám. Jana Palacha 2, 11638 Praha 1, Czech Republic

E-mail address: habicm@ff.cuni.cz

$U R L$ : https://mhabic.github.io 